Check for updates

Cite this: RSC Adv., 2019, 9, 24568

\title{
Current and future functional imaging techniques for post-traumatic stress disorder
}

\author{
Alisha Prasad, (D) $\dagger^{\mathrm{a}}$ Ardalan Chaichi, $\dagger^{\mathrm{a}} \mathrm{D}$. Parker Kelley, (D) $\dagger^{\mathrm{b}}$ Joseph Francis (D) *b \\ and Manas Ranjan Gartia (D) *a
}

Posttraumatic stress disorder (PTSD) is a trauma and stressor related psychiatric disorder associated with structural, metabolic, and molecular alternations in several brain regions including diverse cortical areas, neuroendocrine regions, the striatum, dopaminergic, adrenergic and serotonergic pathways, and the limbic system. We are in critical need of novel therapeutics and biomarkers for PTSD and a deep understanding of cutting edge imaging and spectroscopy methods is necessary for the development of promising new approaches to better diagnose and treat the disorder. According to the Diagnostic and Statistical Manual of Mental Disorders (DSM-V) criterion, all forms of traumatic stress-induced disorder are considered acute stress disorder for the first month following the stressor. Only after symptoms do not remit for one month can the disorder be deemed PTSD. It would be particularly useful to differentiate between acute stress disorder and PTSD during the one month waiting period so that more intensive treatments can be applied early on to patients with a high likelihood of developing PTSD. This would potentially enhance treatment outcomes and/or prevent the development of PTSD. Comprehension of the qualities and limitations of currently applied methods as well as the novel emerging techniques provide invaluable knowledge for fast paced development. Conventional methods of studying PTSD have proven to be insufficient for diagnosis, measurement of treatment efficacy, and monitoring disease progression. As the field currently stands, there is no diagnostic biomarker available for any psychiatric disease, PTSD included. Currently, emerging and available technologies are not utilized to their full capacity and in appropriate experimental designs for the most fruitful possible studies in this area. Therefore, there is an apparent need for improved methods in PTSD research. This review demonstrates the current state of the literature in PTSD, including molecular, cellular, and behavioral indicators, possible biomarkers and clinical and pre-clinical imaging techniques relevant to PTSD, and through this, elucidate the void of current practical imaging and spectroscopy methods that provide true biomarkers for the disorder and the significance of devising new techniques for future investigations. We are unlikely to develop a single biomarker for any psychiatric disorder however. As psychiatric disorders are incomparably complex compared to other medical diagnoses, its most likely that transcriptomic, metabolomic and structural and connectomic imaging data will have to be analyzed in concert in order to produce a dependable non-behavioral marker of PTSD. This can explain the necessity of bridging conventional approaches to novel technologies in order to create a framework for further discoveries in the treatment of PTSD.

Accepted 2nd August 2019

DOI: $10.1039 / c 9 r a 03562 a$ rsc.li/rsc-advances

\section{Introduction}

Post-traumatic stress disorder (PTSD) can be observed in about 20 to $30 \%$ of individuals exposed to extreme traumatic stressors like war, sexual assault, and life-threatening accidents. Despite the fact that about $70 \%$ of individuals experience a serious

\footnotetext{
${ }^{a}$ Department of Mechanical and Industrial Engineering, Louisiana State University, Baton Rouge, LA 70803, USA. E-mail: mgartia@lsu.edu

${ }^{b}$ Comparative Biomedical Sciences, School of Veterinary Medicine, Louisiana State University, Baton Rouge, LA 70803,USA.E-mail:jfrancis@lsu.edu

$\dagger$ Equal contribution authors.
}

trauma in their lifetimes, ${ }^{\mathbf{1}}$ the approximate lifetime occurrence for PTSD is reported to be $6.8 \%$ in the United States and $8 \%$ worldwide. The percentage of individuals that develop PTSD, as opposed to an acute stress response lasting 1-3 weeks is dependent on numerous elements including biological sex and sociocultural factors. An additional point is that according to a recent meta-analysis, unintentional traumas tend to result in a higher percentage of acute PTSD development than do intentional events acutely (30.1\% vs. $11.8 \%$ respectively), but after 12 months, individuals exposed to intentional traumas show a higher percentage of chronic PTSD (14\% vs. $23.3 \%$ respectively). This suggests an interpretive cognitive component 
by the patient..$^{2-4}$ The likelihood of developing PTSD further depends on the intensity of the traumatic event, preexisting stressors experienced by an individual, the number of traumatic events a person is exposed to, and personal resilience and risk parameters. $^{5}$

According to the Diagnostic and Statistical Manual of Mental Disorders (DSM-V) the diagnosis of PTSD should be supported by five diagnostic categories. The first, around which the others must fall is (1) traumatic events: experience to real or vulnerable situations such as, injury, abuse, violence, or death. The DSM-V further requires that at least one symptom from each of the following categories be present for a PTSD diagnosis. (2) Intrusion symptoms: an involuntary re-experiencing of a memory of the event, repeated upsetting thoughts related to past events, or flashbacks associated with the event, intense and extended psychological agony of physiological feedbacks in response to in/external cues or contexts associated with the traumatic incident. (3) Avoidance symptoms: avoidance or efforts to avoid internal or external reminders of the event including distressing memories, feelings, thoughts, people or places associated with the traumatic event. (4) Negative alterations to cognition and mood: including the inability to recall imperative aspects of the trauma, determined and overstated negative theories about oneself, distorted cognitions related to the cause of the event, persistent negative emotional state, reduced interest or contribution in important events, emotional state of disinterest, and/or the inability to experience positive feelings, and (5) marked alterations and reactivity related with the traumatic event, including hypervigilance, irritable behavior and angry outburst, reckless behavior, exaggerated startle response, concentration problems, and sleep disturbances. ${ }^{6}$ PTSD is also associated with substance use disorders, chronic pain, and incapacitating anxiety. ${ }^{5,7,8}$

Traumatic stress can alter gene and therefore protein expression in the brain and periphery, causing downstream alterations to important molecular and neurochemical factors related to affective and cognitive processes, as well as systems involved in coping with and adapting to stress. In addition, stress can alter epigenetics, further modulating gene expression. PTSD is associated with altered immunological factors like increased inflammatory cytokines, particularly IL- $1 \beta$, TNF- $\alpha$, IL6 , and C-reactive proteins. ${ }^{9}$ PTSD is also generally associated with lower baseline levels of glucocorticoids (GC) and impaired hippocampal and prefrontal cortical functioning. ${ }^{10}$ Both psychological factors (cognitive reappraisal and optimism) and biological factors (neurotransmitter systems, single nucleotide polymorphisms (SNPs), epigenetic markers and endocrine factors) are important to the development, modulation, progression, and treatment of PTSD. ${ }^{\mathbf{1 1 , 1 2}}$ Due to the currently limited diagnostic capacity and availability and efficacy of therapy for PTSD, most of the recent research is focused on the identification and detection of potential biomarkers for the disorder. The literature suggests that alterations in neurotransmitter signaling in various brain regions and changes to neuroendocrine signaling are potentially casually related to the development of PTSD and are therefore potential biomarkers for the disorder, but as of now they are limited clinically due to the impossibility of direct measurement in humans in vivo and the low reliability and specificity to PTSD respectively. ${ }^{\mathbf{1 1 , 1 3}}$ Current research should therefore be concentrated on the ability to study these markers in vivo in PTSD patients using novel imaging and spectroscopy techniques, as well as on further elucidating novel cellular and behavioral indicators in animal models. Current studies are focused on identifying changes to receptor densities using positron emission tomography (PET) or single photon emission computed tomography (SPECT), evaluating the regional blood flow using functional magnetic resonance imaging (fMRI), or calculating the regional glucose consumption using PET in PTSD patients compared to healthy individuals or trauma exposed non-PTSD controls. Some brain regions that have been observed to differ in PTSD include, the prefrontal cortex (PFC), insula (Ins), hippocampus (Hipp), orbito-frontal cortex (OFC), parahippocampal regions (pHipp), the amygdala (Amy), the thalamus (Thal), anterior cingulate cortex (ACC), and the hypothalamus (HT).

In this review, we present the current state-of-art of the imaging techniques employed to study PTSD. We highlight the existing imaging and spectroscopy techniques and their advantages and limitations. We also present the emerging techniques and their potential to compensate the deficiencies of current approaches. Fig. 1 presents a schematic showing the organization and highlights of the review.

\section{Background}

\subsection{Molecular, cellular and behavioral indicators in PTSD}

A short list of some currently available cellular and behavioral indicators of PTSD are listed in Fig. 2, and can be divided in four main categories, namely, diagnostic (DSM-V criterion), neuroendocrine, neurochemical and neurotropic indicators, genetic indicators, and immunological indicators respectively. The currently available indicators of PTSD can help in clinical diagnosis and therapy although they are currently not specific enough for effective diagnosis, medication choice, or therapy for PTSD based on the indicators alone. ${ }^{14}$ Hence, there is a need at present to find novel biomarkers for diagnosis and to determine which treatments will be most effective for treating PTSD. ${ }^{15}$ Resilience markers should also be taken into consideration due to the remarkable population of people who are exposed to traumatic stressors and do not develop PTSD. ${ }^{12,13}$

\subsection{Neurotransmitters in PTSD}

The only approved pharmacotherapies for PTSD are SSRI antidepressants. Particular selective serotonin reuptake inhibitors (SSRIs) like paroxetine and sertraline significantly improve symptoms in about $50-60 \%$ of PTSD patients, but this is not that much greater than the effect of placebo, which is about $40 \%$. Furthermore, it must be acknowledged that a headache is not the absence of Advil, as the common saying goes. The partial efficacy of SSRIs in depression and PTSD does not necessarily suggest that there is a problem with serotonin (5HT) in these disorders, only that altering 5-HT signaling can improve symptomology. ${ }^{16}$ Decades of study on 5-HT however 


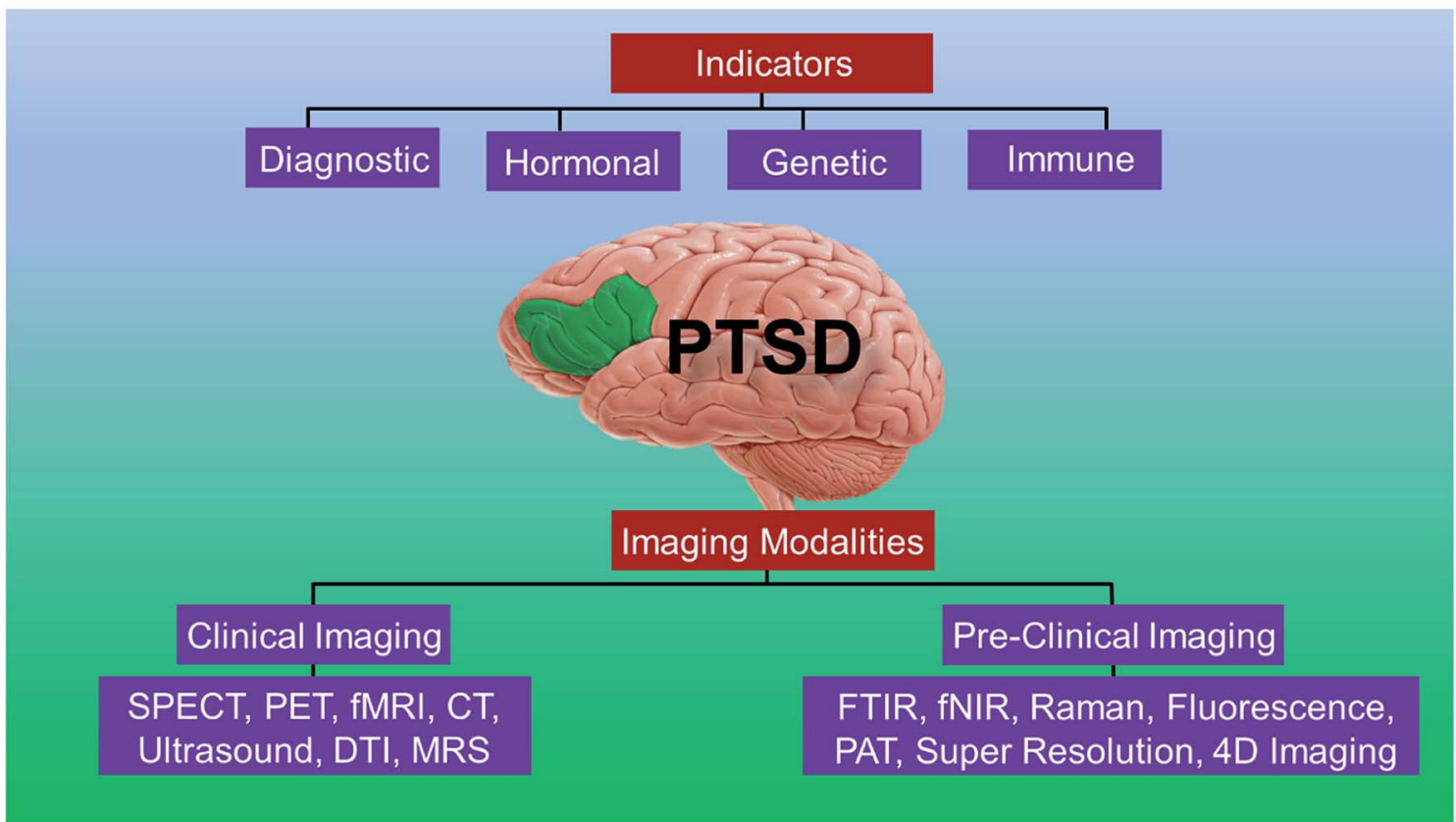

Fig. 1 Schematic showing the organization and highlights of the review.

suggest a role of 5-HT in several processes involved in PTSD, particularly in coping with or adapting to stress, sleep, memory, aggression, social bonding and general cognitive processes. ${ }^{17,18}$
It is currently impossible to measure 5-HT in the brain of live humans, although it can be measured indirectly through the measurement of 5-HT-related receptors, transporters, enzymes

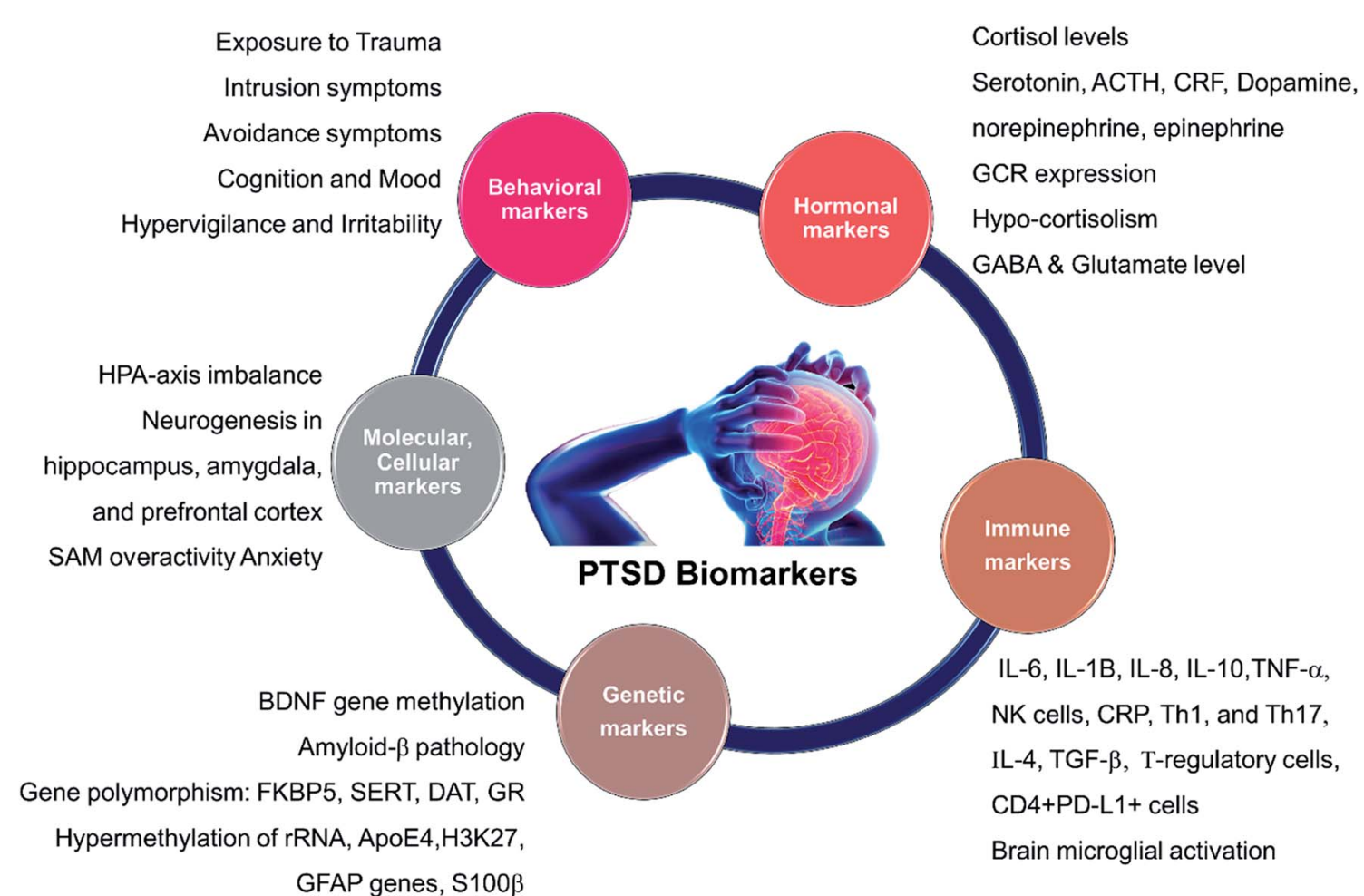

Fig. 2 Different biomarkers of PTSD. HPA: hypothalamic pituitary adrenal; SAM: Synergistic Activation Mediator; ACTH: adrenocorticotropic hormone; CRF: corticotropin hormone releasing factor; GCR/GR: glucocorticoid receptor; BDNF: brain-derived neurotrophic factor; GABA: Gamma-Aminobutyric Acid; FKBP5 (protein); SERT: serotonin transporter or 5-HTT; DAT: dopamine transporter gene; rRNA: ribosomal ribonucleic acid; ApoE4: apolipoprotein E; H3K27: trimethylated histone H3 lysine 4 amino acid; GFAP: glial fibrillary acidic protein; IL: interleukin; TNF: tumor necrosis factor I; NK: Natural Killer cells; Th: T-helper cells. 
and by measuring peripheral 5-HT and 5-HT metabolites. These methods are limited however since clinical investigators cannot control PTSD development in a population of genetically similar individuals, as can be done in animal studies.

Dr Joseph Francis' Lab at LSU used a predator exposure model of PTSD in rats to show that total 5-HT levels in the PFC and Hipp are decreased in PTSD animals compared to control animals. We also measured total norepinephrine (NE) levels and observed that NE levels were increased in the same regions. ${ }^{19}$ These data indicate that neurotransmitter changes in PTSD may be causally related to the disorder rather than being preexisting or predispositional. Future studies in this area could use molecular imaging techniques, like PET and SPECT imaging to investigate alterations to 5 - $\mathrm{HT}$ and NE binding and receptor expression levels in military veterans before and after combat to determine if these changes exist in humans and to study their time course throughout the disorder and between combat exposed individuals that do and do not develop PTSD. Monoamine neurotransmitter levels themselves cannot be observed in living humans, but a proxy for extracellular neurotransmitter levels in humans could be the use of competitive binding studies with known 5-HT and NE agonists/antagonists bound to radionucleotides through PET or SPECT imaging.

\subsection{Genetic and epigenetic considerations}

This section is offered as a partial guide to potential biomarkers in PTSD and to offer several recent findings on the subject which will inform future imaging investigations. These include a model of genetic predispositions to the development of PTSD and depression and the potential for an interaction with epigenetic changes associated with childhood trauma, possibly further tipping the scales toward susceptibility to psychiatric disorder. Beyond the description of an important aspect of the most recent PTSD literature, glucocorticoids, this section will also offer a jumping off point for future investigations that seek to integrate imaging findings with the most up to date findings in the molecular and genetic underpinnings of PTSD.

Epigenetic alterations can result from experience or be inherited inter-generationally. In psychiatric disorders, epigenetic changes associated with early life adversities are of particular concern. Epigenetic changes can be measured directly through the analysis of methylation patterns on DNA or identified by modification of gene expression. Alterations to the transcriptome can be messenger RNA (mRNA) levels transcribed from these genes by PCR, in situ hybridization, or transcriptome sequencing. Transcriptomic changes in the brain can only be studied in animal models at the moment, but investigators also study changes to peripheral mRNA expression in search for relevant biomarkers in PTSD. The brain regions typically studied for epigenetic changes resulting from or predisposing one to PTSD are shown in Fig. 3(a). ${ }^{20}$

Genetic and epigenetic alterations in PTSD are often evaluated in relation to the hypothalamic pituitary adrenal (HPA) axis. Corticotrophin Releasing Hormone (CRH) and Arginine Vasopressin (AVP) are two hormones that control the behavior and metabolism in response to stress. CRH, released from the hypothalamus in response to stress/trauma causes the release of adrenocorticotropin releasing hormone (ACTH) from the pituitary gland which in turn causes cortisol (CORT) release from the adrenal gland, which feeds back to the hypothalamus in a negative feedback loop, thus reducing CRH expression. CORT is a glucocorticoid (GC) and thought to be the major stress hormone in humans. GC signaling is complex, involving chaperon proteins, transcription factors and signaling cascades of accessory proteins which all help to restrict stress hormone expression by the negative feedback mechanism discussed above. ${ }^{21}$ GC help biological organisms to cope with the stress by facilitating resource utilization and reducing inflammation, but can also cause serious consequences if they persist at high levels for too long.

A greater understanding of the genetic and epigenetic risk factors associated with HPA axis function in PTSD and depression may help us elucidate causal mechanisms involved in these disorders. Fig. 3(b) shows a model to understand the epigenetic features responsible for depression and PTSD. The first arrow indicates that a predisposition to developing PTSD could be due to genetic mutations (red DNA double helix), which can later manifest as depression and/or PTSD development (red flash), but in the absence of trauma, they can still lead a healthy life as shown (orange colored child). The red flash on the second arrow indicates that childhood difficulties eventually leads to an altered translation of genes via DNA (de-)methylation, an epigenetic alteration, which may increase chances of PTSD development (red colored lolly on the helix). The third arrow indicates that early traumatic events can cause epigenetic alterations via DNA (de-) methylation, which can interact with existing mutations, possibly resulting in PTSD and depression (orange DNA becomes red DNA with red lolly). This model supports the concept that an infant carrying a stress allele is more likely to experience deleterious epigenetic changes associated with that gene. For example, the gene FKBP5 is associated with glucocorticoid signaling and the risk allele for that gene is associated with a PTSD diagnosis, as is childhood abuse. ${ }^{22}$ This model suggests that risk alleles, like FKBP5, could be triggered by childhood stress or trauma through epigenetic changes to gene expression and lead to the progression of PTSD and depression. ${ }^{23}$

Additionally, to their role in the stress response, GCs are also involved in the negative feedback mechanism controlling stress-associated immune activation, particularly through the inhibition of a transcription factor called NF-kB. Low baseline GCs expression is already known to be a potential biomarker for PTSD and PTSD is known to be associated with excessive immune activation. ${ }^{24,25}$ Furthermore, PTSD is associated with reduced sensitivity to GC. Recently, a study examined the role of GCs in PTSD by conducting a survey of 52 male and female veterans with PTSD who received either prolonged exposure (PE) treatment or a weekly minimal attention (MA) involvement for 12 consecutive weeks. The study did not show any effect of treatment on the clinician administered PTSD scale (CAPS). The lysozyme inhibition test was conducted with cultured peripheral blood mononuclear cells along with variable doses of dexamethasone (DEX), an anti-inflammatory synthetic steroid 


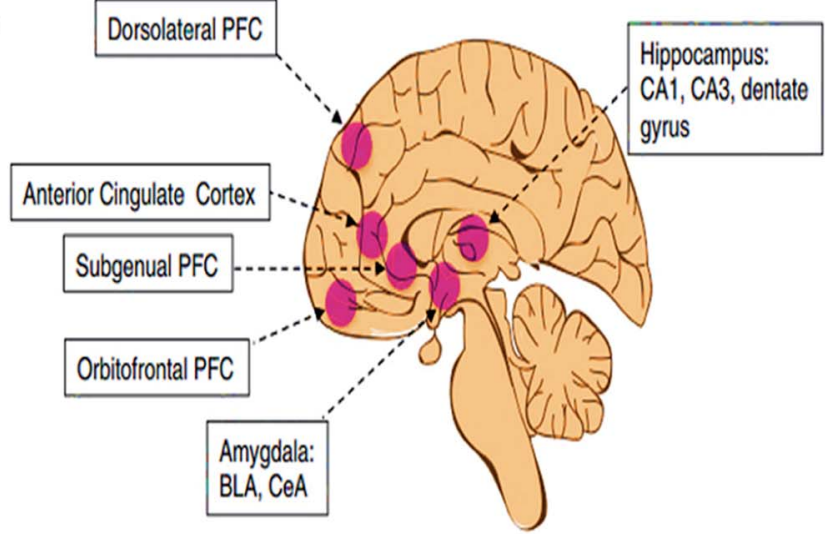

C

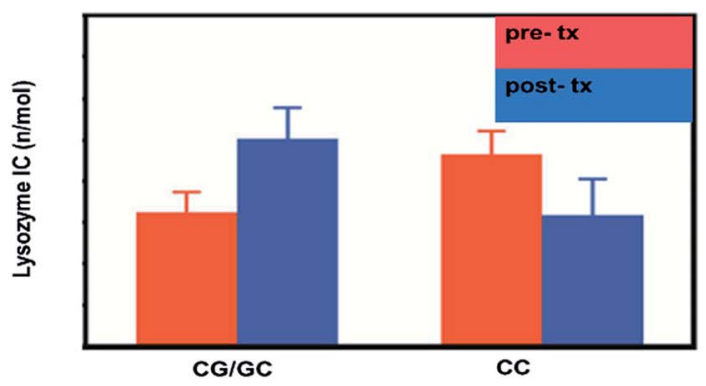

b

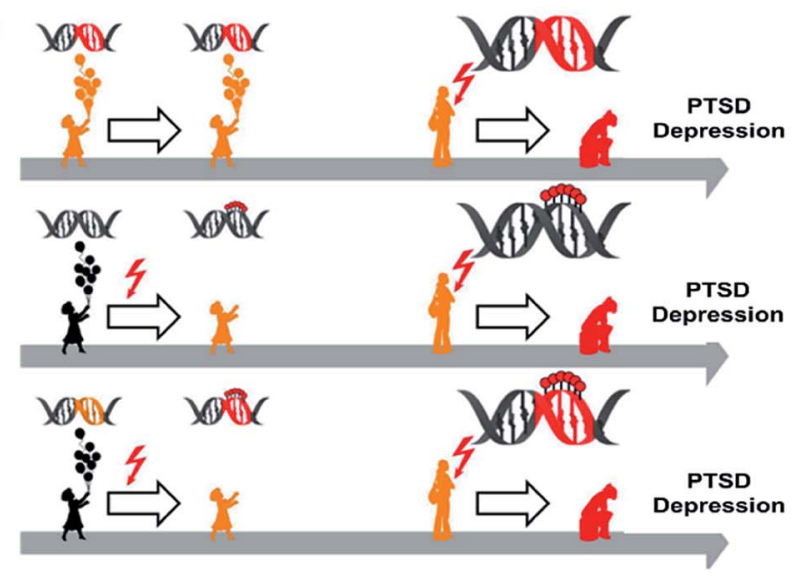

d

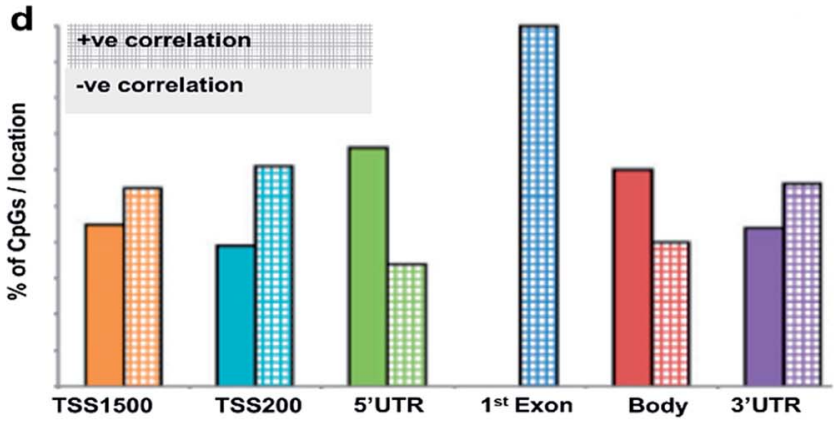

Fig. 3 Epigenetic considerations. (a) Regions of brain involved in PTSD. Reproduced with permission from ref. 20. Copyright 2017, Society of Biological Psychiatry. (b) Mechanism of action of PTSD. Reproduced with permission from ref. 23. Copyright 2013, Raabe and Spengler. (c) Glucocorticoid sensitivity at pre- and post-treatment depending on GR BCLI polymorphism genotype (CG/GC or CC). Data were adjusted for age, gender and body mass index and are represented as mean \pm SEM. Statistical significance was set at $p<0.05$. Reproduced with permission from ref. 26. Copyright 2014, Published by the Royal Society. (d) Correlations of DNA methylation with gene expression. The bar graph shows the percent of CpGs with positive vs. negative correlations with gene expression. Reproduced with permission from ref. 31. Copyright 2013, National Academy of Sciences.

mimicking the effects of endogenous GC, to measure the GCs sensitivity (Fig. 3(c)). GCs sensitivity at pre-and post-treatment was dependent on the patient's glucocorticoid receptor gene GR BCLI genotype. Patients either had the wild-type (CC) or heterozygous genotype (CG/GC). Chi-squared analysis confirmed that patients were more likely to carry the GG/GC ( $\sim 71 \%)$ than the CC genotype ( $\sim 30 \%)$. GC sensitivity depended on the patient's genetic status and genetics predicted the treatment response such that those with GG/GC phenotype showed a decrease in GC sensitivity post treatment while those with CC genotype did not. These data suggest that genetics associated with the GC system can be helpful in understanding the mechanisms of action underlying symptom reduction and glucocorticoid sensitivity in PTSD. ${ }^{26}$

Previous reports have found that GC hormones are elevated in severely depressed patients ${ }^{27}$ and generally found to be reduced in PTSD, although there is some debate on the matter. ${ }^{28}$ Literature reveals that high GCs can reduce hippocampal neurogenesis and hinder the growth of neurons from stem cells in animal models. ${ }^{29,30}$

Elisabeth Binders Lab at the Max Plank Institute also studied the impact of childhood maltreatment by evaluating the epigenetic modifications in PTSD versus healthy patients. The
DNA transcripts with CpG island were evaluated for genetic differences. It was found that $69.3 \%$ of transcripts with at least one CpG island were identified for PTSD due to genetic mutations at adolescence/childhood difficulties, whereas only $33.6 \%$ were identified for other forms of PTSD (Fig. 3(d)). Methylation in the CpGs island are more significant at $3^{\prime} \mathrm{UTR}$ region emphasizing the importance of epigenetic factors to differentiate the types of PTSD. ${ }^{31}$

Collectively these data highlight the importance of the GC system in PTSD and the related genetic and epigenetic differences that could impact disease development, treatment response, and outcome. Future imaging studies in this area should further investigate the functional (fMRI, PET, EEG) and molecular differences (PET, SPECT) in PTSD related brain regions, like the Hipp and pHipp, which have high levels of the glucocorticoid (GR) and mineralocorticoid receptors (MR), and determine if there is any association between these functional and molecular differences and a patient's genotype, childhood trauma, peripheral GC levels, and PTSD symptoms. These investigations should further utilize special experimental designs like twin studies and comparisons between PTSD patients and trauma exposed controls to further elucidate whether changes to the GC system precede or are caused by PTSD. 


\subsection{Imaging in PTSD: what do we know?}

Although much of the underpinnings of PTSD are still to be uncovered, there do now exist many highly reported features of PTSD that are accessible through commonly available neuroimaging modalities. These features fall into several major categories including structural differences in various brain regions, functional differences within and between various brain regions and networks and molecular and neurochemical differences, which are further divided by the particular systems that those factors play a role in governing. Comparisons will be presented as either between PTSD patients and healthy controls or else between PTSD patients and combat exposed and/or trauma exposed entities who did not go on to develop PTSD.

Five major brain regions represent the core of PTSD research, but that list is quickly expanding and each region is additionally being parsed into more and more granular subregions with distinct functionalities. We will predominantly review only the major regions here, but in some cases, we may include information on other regions if the weight of evidence is substantial enough to warrant it. These major regions are the hippocampus (Hipp) and parahippocampal regions (Hipp), known more generally as the medial temporal lobe (MTL), the ventromedial prefrontal cortex (vmPFC), the amygdala (Amy), the anterior cingulate cortex (ACC), and the insula (Ins). ${ }^{32-34}$

The purpose of this part of the communication is to provide a global perspective on the structural and functional basis of PTSD and to explicate the methods by which we may begin to understand the molecular basis of these functional changes.

2.4.1. Structural differences in PTSD. The major technique used to measure structural changes in PTSD and other neurological disorders is MRI. The most well-known structural difference observed in PTSD is the bilateral reduction in Hipp volume when equated against both fit controls and trauma exposed controls. This difference is observed when analyzing either the right Hipp, the left Hipp or both sides in combination. ${ }^{33}$ The Hipp is critical for new memory formation, working memory function, contextual processing, and updating existing memory formations with new data and contexts. As part of the limbic system, it also plays a major role in affective processing, spatial navigation, and autobiographical memory. The Hipp, along with the rest of the MTL, is also a major hub of the default mode network (DMN), a major network involved in selfreferential and task negative processing. ${ }^{35}$ Despite a wealth of evidence suggesting smaller hippocampal volumes in PTSD patients, numerous studies have also reported that no significant volumetric differences exist. ${ }^{33,36}$

Another consistent structural difference observed in PTSD is in the anterior cingulate cortex. The ACC is a cortical region layered above the corpus callosum (CC) and has two functionally distinct regions. The rostral-ventral region closest in proximity to the CC is implicated in emotional processing and is often called the limbic cortex. The dorsal component is the cognitive division and is involved in response inhibition to nonemotional stimuli. Strong evidence now exists suggesting the ACC plays a role in conflict monitoring, cognitive dissonance, and the subsequent adjustments of brain activity required for processing conflicting information. ${ }^{37,38}$ Numerous studies have exhibited reduced gray matter in the ACC in PTSD compared to both trauma exposed controls ${ }^{32,39}$ and healthy control participants. ${ }^{34}$

The amygdala, like the Hipp and ACC, is a component of the limbic system. The Amy is crucial for fear and stress-related memory formation including fear conditioning ${ }^{40}$ and generalization, ${ }^{41}$ and the resultant fear and emotionally related behaviors in response to reminders of the trauma. ${ }^{42}$ Reports on structural differences in the amygdala (Amy) in PTSD are mixed, some reporting reduced Amy volumes ${ }^{43}$ in PTSD patients and others suggesting either non-significantly increased volumes ${ }^{\mathbf{4 4}}$ or no differences between PTSD patients and control participants. $^{43}$ A meta-analysis including only a very homogenous group of participants in just three studies $(N=141)$ has however exhibited smaller Amy volumes in PTSD vs. healthy controls and traumatized non-PTSD controls.

If these structural differences in the limbic regions of PTSD patients do stand the test of time, it begs the question as to whether they precede or are caused by a traumatic event. Some of the most compelling evidence on this front has come from twin studies performed by the lab of Roger K. Pitman at Massachusetts General Hospital. These investigators compared combat exposed monozygotic twins who had either developed $(N=18)$ or did not develop $(N=23)$ PTSD in the Vietnam war with their combat-unexposed cotwins. Combat exposed twins with PTSD exhibited significantly reduced gray matter densities in the right Hipp, the pregenual ACC and both the left and right insula compared to their combat unexposed co-twins. ${ }^{45}$ This indicates that the differences are most likely caused by PTSD rather than the other way around. These data highlight the importance of between-participant controls on the measurement of structural changes associated with PTSD.

2.4.2. Functional differences in PTSD. There is a wealth of information available on the differences in regional activity within different experimental paradigms in the study of PTSD in Table 2. These data include blood oxygen level dependent (BOLD) measures from fMRI, regional cerebral blood flow and glucose utilization data measured by PET and SPECT imaging, and molecular data as also measured through PET and SPECT. These data alone, while meaningful, are not of sufficient explanatory value to offer a complete understanding of the neurophysiology of PTSD, but recently, functional neuroimaging investigations of PTSD are shifting towards a network based perspective.

Network based neurobiological models of psychiatric disorders are the new frontier of integration between cognitive psychology and biomedical neuroscience. An important concept when considering imaging studies on psychopathology is functional connectivity. This refers to a statistical (Pearson correlation or Granger causality) coupling or association within or between brain regions of interest (ROI). Functional connectivity is essentially the basis of network based modeling. Recently, evidence is accumulating that suggests the brain is composed of functionally differentiated intrinsic connectivity networks (ICNs). These networks provide a broader framework in which to understand neurobiological dysfunction in PTSD 
rather than being limited to the interactions of discrete structures. ${ }^{35}$

There are 3 major networks commonly implicated in PTSD. These include the default mode network (DMN), also known as the task negative network, which is composed of the medial temporal lobe (MTL), the ventromedial prefrontal cortex (vmPFC), and the posterior cingulate cortex (PCC), the salience network (SN), also known as the ventral attention network, which is composed by the anterior cingulate cortex (ACC), the amygdala (Amy), and the insula (Ins) and the central executive network (CEN), also known as the fronto-parietal control network, which is composed of the dorsolateral prefrontal cortex (dlPFC) and the precuneous. ${ }^{35,46}$

The DMN is most active in normal control participants during task negative self-reflection. In PTSD, the DMN exhibits decreased coupling between each of these components at rest and this dysconnectivity correlates with symptom severity. Despite limited studies on the CEN in PTSD, there is some evidence of reduced functional connectivity in the CEN including the reduced ability of the CEN to recruit the precuneus during an autobiographical memory task. ${ }^{47}$ The salience network modulates attentional focus and the initiation of homeostatic responses to environmental events that are critical for survival. It does this through bottom up information integration and subsequent communication with higher order centers of cognition about the importance of environmental information. The SN is a sort of alarm system capable of triggering hypervigilance, irritability and aggression, a few diagnostic criterion of PTSD. The SN also plays a role in switching between the DMN and various task positive networks. In PTSD, the $\mathrm{SN}$ is typically over active both in its individual components across various tasks, but also in the functional connectivity between them. ${ }^{35,46}$ Overall, PTSD patients tend to exhibit increased salience processing, resulting in hypervigilance along with disrupted DMN integrity and an underactive CEN, resulting in poor top down control, poorly organized self-referential processing and dysregulated transitions between networkstates. An extension of this literature would be a detailed mapping of particular PTSD symptom clusters to these intrinsic brain networks as is currently being performed by some labs. ${ }^{48}$ This sort of mapping could potentially be used in the future for diagnosis and confirmation of particular PTSD phenotypes and further articulated through a molecular imaging analysis of the underlying molecular changes which substantiate these functional differences.

Despite these substantial advances in understanding the functional variances among PTSD patients and trauma exposed or fit controls, we must always take into account that the underpinnings of these changes are molecular, neurochemical, and potentially neurotropic and structural. The observed differences in network integrity and function are based in molecular changes at the neuronal level that can currently only be measured in animal models and theoretically measured, albeit much more slowly, in human patients through molecular imaging. Molecular imaging through PET and SPECT are currently extremely limited in comparison to what can be done in animal models. In animal models, techniques like whole transcriptome RNA sequencing, metabolomics, proteomics, and lipidomics can give us a broad view of what changes are caused by trauma. One frailty of each of these techniques however is that they can only be performed in humans and animals post mortem. This makes it difficult to show causation within a single individual. Novel techniques, like Raman spectroscopy, that will be introduced later in this paper, can offer real time monitoring of molecular changes in the brain of animal models of PTSD and someday, this and/or similar techniques may be available for human research.

The rest of this paper will focus on the scientific basis of current and future imaging modalities applicable to PTSD, with a specific focus on molecular imaging in humans and other techniques, like Raman spectroscopy, and Fourier-transform infrared (FTIR) imaging by which we can investigate the molecular basis of these functional changes in animal models and ex vivo samples, setting the groundwork for the eventual technological progress necessary to monitor these changes noninvasively in human patients.

\section{Imaging techniques}

\subsection{Clinical imaging techniques}

Recently, functional neuroimaging methods have been utilized in neurological studies of various diseases to elucidate their mechanisms. SPECT, PET and fMRI are three fundamental techniques that measure physiological criteria like molecular factors (PET, SPECT), cerebral blood flow to specific regions of the brain over time (fMRI) and regional energy consumption via glucose utilization (PET), to investigate brain functionality. These techniques measure indirect neural activity based on the fact that neural activation requires blood flow, metabolism, and neurotransmission to occur. fMRI illustrates brain activity through Blood Oxygen Level Dependent (BOLD) changes. On other hand, PET and SPECT take advantage of regional cerebral blood flow (rCBF) and neuroreceptor concentrations to demonstrate patterns of brain activity and function. ${ }^{10,49} \mathrm{~A}$ summary of the imaging modalities currently used for PTSD study are provided in Table 1 and the main findings with the contrast agents used are provided in Table 2.

3.1.1. Single-photon emission computed tomography (SPECT). The SPECT technique visualizes the blood flow in the brain, periphery, and other regions of the cerebrum through the injection of a radio tracer labeled with a bioactive molecule, which provides the ability to measure the target of interest. Tc99 is a commonly used radiotracer which emits $\gamma$ particles that provide the signal from which to measure alternations in the brain. A $\gamma$ camera receives single $\gamma$ photon signals from the radiotracer and provides data from which to build an image. A significant drawback of this method is the lack of positional information due to the emission of a single photon at a time. To overcome this issue, the travelling of photons in a particular direction should be measured by a lead collimator. Hexamethyl propylene-amine-oxime (HMPAO) labeled Tc-99m is the most prevalent $\gamma$ emitter used in this method, along with sodium iodide (NaI) crystals as rCBF detectors. Other radiotracers, like iomazenil [I-123], which measures the GABA receptor 
Table 1 Summary of imaging modalities ${ }^{a}$

\begin{tabular}{|c|c|c|c|c|c|c|c|}
\hline Technique & Depth & $\begin{array}{l}\text { Spatial } \\
\text { resolution }\end{array}$ & $\begin{array}{l}\text { Temporal } \\
\text { resolution }\end{array}$ & Strength & Limitation & $\begin{array}{l}\text { Clinical } \\
\text { use }\end{array}$ & Physical principle \\
\hline SPECT & $\begin{array}{l}\text { Whole } \\
\text { brain }\end{array}$ & $1-5 \mathrm{~mm}$ & Minutes & $\begin{array}{l}\text { Non-invasive, lower cost } \\
\text { compared to PET, longer } \\
\text { half-life }(\sim 6 \mathrm{~h}) \text { for radio } \\
\text { tracers, allow longer } \\
\text { imaging time, sensitivity: } \\
10^{-10} \text { to } 10^{-11} \mathrm{~mol} \mathrm{~L}^{-1}\end{array}$ & 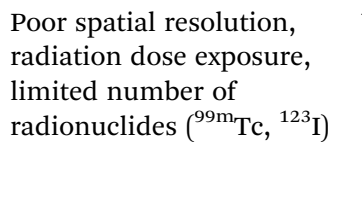 & Yes & Low-energy $\gamma$-rays \\
\hline PET & $\begin{array}{l}\text { Whole } \\
\text { brain }\end{array}$ & $1-5 \mathrm{~mm}$ & $\begin{array}{l}\text { Seconds to } \\
\text { minutes }\end{array}$ & $\begin{array}{l}\text { High sensitivity compared } \\
\text { to SPECT, radionuclides } \\
\text { used in PET }\left({ }^{11} \mathrm{C},{ }^{13} \mathrm{~N},{ }^{8} \mathrm{~F}\right) \text { are } \\
\text { abundant in the body and } \\
\text { can be tailored for } \\
\text { endogenous biomolecules } \\
\text { such as carbohydrates, fats, } \\
\text { nucleic acids and proteins, } \\
\text { sensitivity: } 10^{-11} \text { to } \\
10^{-12} \mathrm{~mol} \mathrm{~L}^{-1}\end{array}$ & $\begin{array}{l}\text { Short half-life }(\sim 75 \mathrm{~s}) \text { for the } \\
\text { radio tracers and hence } \\
\text { must be produced onsite } \\
\text { before imaging, high-cost } \\
\text { compared to SPECT, limited } \\
\text { imaging time window }\end{array}$ & Yes & High-energy $\gamma$-rays \\
\hline CT & $\begin{array}{l}\text { Whole } \\
\text { brain }\end{array}$ & $50 \mu \mathrm{m}$ & Minutes & $\begin{array}{l}\text { High spatial resolution, fast } \\
\text { and cross-sectional images } \\
\text { of the brain, sensitivity: } \\
10^{-6} \mathrm{~mol} \mathrm{~L}^{-1}\end{array}$ & $\begin{array}{l}\text { Structure of the brain, not } \\
\text { its function, low contrast }\end{array}$ & Yes & X-rays \\
\hline fMRI & $\begin{array}{l}\text { Whole } \\
\text { brain }\end{array}$ & $25-100 \mu \mathrm{m}$ & $\begin{array}{l}\text { Minutes to } \\
\text { hours }\end{array}$ & $\begin{array}{l}\text { No radiation, structural and } \\
\text { functional data, high spatial } \\
\text { resolution, greater contrast } \\
\text { for soft tissues, imaging } \\
\text { agents with lower toxicity, } \\
\text { sensitivity: } 10^{-3} \text { to } \\
10^{-5} \text { mol L }^{-1}\end{array}$ & $\begin{array}{l}\text { High cost, long scanning } \\
\text { time, sensitive to motion } \\
\text { artifacts, relatively low } \\
\text { sensitivity and low contrast }\end{array}$ & Yes & Radio waves \\
\hline Ultrasound & $1-5 \mathrm{~cm}$ & $50-500 \mu \mathrm{m}$ & $\begin{array}{l}\text { Seconds to } \\
\text { minutes }\end{array}$ & $\begin{array}{l}\text { Low cost, no radiation, high } \\
\text { speed, portable, sensitivity } \\
\text { with microbubbles: } \\
10^{-12} \mathrm{~mol} \mathrm{~L}^{-1}\end{array}$ & Low contrast & Yes & $\begin{array}{l}\text { High frequency } \\
\text { sound waves }\end{array}$ \\
\hline EEG & Scalp & $5-10 \mathrm{~cm}$ & Millisecond & $\begin{array}{l}\text { Inexpensive, portable, high } \\
\text { temporal resolution, } \\
\text { electrical activity of brain }\end{array}$ & $\begin{array}{l}\text { Low spatial resolution, } \\
\text { prone to error due to } \\
\text { environmental noise, } \\
\text { localization of signal is } \\
\text { difficult }\end{array}$ & Yes & Electrical \\
\hline MEG & Scalp & $1-5 \mathrm{~mm}$ & Millisecond & High temporal resolution & Expensive & Yes & Electromagnetic \\
\hline fNIR & $<1 \mathrm{~cm}$ & $2-3 \mathrm{~mm}$ & $\begin{array}{l}\text { Seconds to } \\
\text { minutes }\end{array}$ & $\begin{array}{l}\text { No radiation, inexpensive, } \\
\text { sensitivity: } 10^{-9} \text { to } \\
10^{-12} \mathrm{~mol} \mathrm{~L}^{-1}\end{array}$ & $\begin{array}{l}\text { Scattering due to tissues } \\
\text { may be a problem, low } \\
\text { penetration depth }\end{array}$ & Preclinical & Near-infrared light \\
\hline Photoacoustic & $\begin{array}{l}0.6-5 \\
\mathrm{~cm}\end{array}$ & $\begin{array}{l}10 \mu \mathrm{m} \text { to } 1 \\
\mathrm{~mm}\end{array}$ & $\begin{array}{l}\text { Seconds to } \\
\text { minutes }\end{array}$ & $\begin{array}{l}\text { No radiation, label-free, } \\
\text { high spatial resolution and } \\
\text { low cost compared to CT/ } \\
\text { PET, sensitivity: } 10^{-6} \text { to } \\
10^{-12} \text { mol L }^{-1}\end{array}$ & $\begin{array}{l}\text { Distortion of acoustic signal } \\
\text { due to skull, temperature } \\
\text { dependent signal, weak } \\
\text { absorption at shorter } \\
\text { wavelengths }\end{array}$ & Preclinical & $\begin{array}{l}\text { Pulsed laser and } \\
\text { sound wave }\end{array}$ \\
\hline VSDI & $1 \mathrm{~mm}$ & $50 \mu \mathrm{m}$ & Millisecond & High temporal resolution & $\begin{array}{l}\text { Invasive, prone to } \\
\text { photobleaching of dye, toxic } \\
\text { to cells }\end{array}$ & Preclinical & Voltage sensitive dye \\
\hline Bioluminescence & $1-2 \mathrm{~cm}$ & $3-5 \mathrm{~mm}$ & $\begin{array}{l}\text { Seconds- } \\
\text { Minutes }\end{array}$ & $\begin{array}{l}\text { No radiation, high } \\
\text { sensitivity, inexpensive, } \\
\text { sensitivity: } 10^{-15} \text { to } \\
10^{-17} \text { mol L }\end{array}$ & $\begin{array}{l}\text { Scattering due to tissues } \\
\text { may be a problem, spatial } \\
\text { resolution is low }\end{array}$ & Preclinical & Visible light \\
\hline LSCI & $\begin{array}{l}0.5-1 \\
\mathrm{~mm}\end{array}$ & $10 \mu \mathrm{m}$ & Microseconds & $\begin{array}{l}\text { Label-free, high temporal } \\
\text { resolution }\end{array}$ & Invasive & Preclinical & $\begin{array}{l}\text { Visible and near } \\
\text { infrared laser }\end{array}$ \\
\hline Two-photon & $1 \mathrm{~mm}$ & $1 \mu \mathrm{m}$ & Microseconds & High spatial resolution & $\begin{array}{l}\text { Invasive, photobleaching } \\
\text { issues with dyes, scattering } \\
\text { due to tissues }\end{array}$ & Preclinical & Infrared laser \\
\hline FTIR & $<1 \mathrm{~cm}$ & $5-12 \mu \mathrm{m}$ & $\begin{array}{l}\text { Seconds to } \\
\text { minutes }\end{array}$ & $\begin{array}{l}\text { Label-free method, short } \\
\text { imaging time }\end{array}$ & $\begin{array}{l}\text { High attenuation in liquid } \\
\text { environment, difficult to } \\
\text { distinguish closely related } \\
\text { molecular structures }\end{array}$ & Preclinical & Infrared light \\
\hline
\end{tabular}


Table 1 (Contd.)

\begin{tabular}{|c|c|c|c|c|c|c|c|}
\hline Technique & Depth & $\begin{array}{l}\text { Spatial } \\
\text { resolution }\end{array}$ & $\begin{array}{l}\text { Temporal } \\
\text { resolution }\end{array}$ & Strength & Limitation & $\begin{array}{l}\text { Clinical } \\
\text { use }\end{array}$ & Physical principle \\
\hline Raman & $5 \mathrm{~mm}$ & $<1 \mu \mathrm{m}$ & $\begin{array}{l}\text { Minutes to } \\
\text { days }\end{array}$ & $\begin{array}{l}\text { Label-free analysis, high } \\
\text { spatial resolution, can work } \\
\text { with liquid environment }\end{array}$ & $\begin{array}{l}\text { Complex statistical analysis } \\
\text { may be required to separate } \\
\text { analytes, long imaging time } \\
\text { required for imaging large } \\
\text { area at high resolution }\end{array}$ & & $\begin{array}{l}\text { Visible and near } \\
\text { infrared laser }\end{array}$ \\
\hline
\end{tabular}

${ }^{a}$ SPECT: Single Photon Emission Computed Tomography; PET: Positron Emission Tomography; CT: Computed Tomography; fMRI: functional Magnetic Resonance Imaging; EEG: electroencephalogram; MEG: magnetoencephalography; fNIR: functional Near-Infrared Imaging; VSDI: Voltage-Sensitive Dye Imaging; LSCI: Laser Speckle Contrast Imaging; FTIR: Fourier Transform Infrared Microscopy.

benzodiazepine binding site are also utilized in SPECT. In comparison with other methods like PET, SPECT has a simpler methodology and it is accessible in almost all the nuclear medicine facilities. Furthermore, there is no need for on-site production of radionuclides, since the $\gamma$ emitter materials in SPECT has a long half-life, compared to most PET tracers. Moreover, this method is quite affordable due to the reasonable costs of primary substances and equipment. SPECT however, has numerous disadvantages as well. Apart from the lack of positional information in SPECT, the sensitivity and spatial resolution of this method are considerably lower than other methods and this creates limitations for the investigation of deeper regions of the brain. This restriction is caused by signal attenuation from tissues between the energy source and detector. Some mathematical approaches have been utilized to compensate for signal attenuation and increase accuracy. However, these approaches do not make significant enough corrections for deep brain activity scanning. Furthermore, radionuclide tagged biomolecules like [Tc-99m] HMPAO, usually have different behaviors than native molecules that would cause complicated issues for data analysis. Finally, SPECT is considered an invasive method that can be detrimental for human health due to the injection of radionuclides into the body. ${ }^{\mathbf{1 0 , 5 0}}$

The efficacy of SPECT is dependent on the biomolecule associated with the radio tracer. This allows SPECT to be utilized in numerous applications, only limited by the radiotracers available. In addition to 99mTc-HMPAO, 99mTc-ECD also measures cerebral blood flow. As opposed to large research projects, SPECT is also used to investigate individual cases in war torn areas like Iran. For example, SPECT imaging of a 54 year old male with PTSD was studied by Majid Assadi's Lab at the Kasra Hospital in Tehran, Iran, showing that hypoperfusion is observed in the left inferior temporal region of his brain. ${ }^{51}$

In a recent review by Raji et al. at $\mathrm{UCSF}^{52}$ the authors elaborated the ability of SPECT imaging to study a similar and often comorbid disease to PTSD, mild traumatic brain injury (mTBI), in human subjects. Accordingly, SPECT can be a promising method of investigating mTBI when equipped with quantitative analysis techniques. Their results indicate that when rCBF is measured in mTBI patients by SPECT imaging, abnormal frontal lobe perfusion was indicated in 94\% of studies. Additionally, perfusion abnormalities have been detected by the SPECT approach in temporal (76\%), parietal (71\%), occipital $(53 \%)$ and cerebellar $(23 \%)$ regions of brain. ${ }^{52}$

In the characterization of a new SPECT ligand, Fabio et al. suggested SRX246, a vasopressin V1a antagonist has the ability to pass through the blood brain barrier of rats. Due to its high selectivity and permeability, SRX246 can be utilized in the radio labeling of V1a receptors in various stress related disorders. This new molecule remarkably improves characterization and visualization of SPECT imaging method for vasopressin receptors. $^{53}$

In another clinical study, Hoexter et al. investigated the dopaminergic system in vivo in PTSD patients by means of SPECT imaging, using 99mTcTRODAT-1, a ligand for the dopamine transporter (DAT). They concluded that the density of striatal DAT is elevated in PTSD compared controlled subjects. ${ }^{54}$ Recently, thirty military subjects were studied by Harch et al. by means of clinical medicine and SPECT imaging. The main goal of their study was investigating the effectiveness and feasibility of hyperbaric oxygen treatments (HBOT) for PTSD and mild TBI persistent post-concussion syndrome (PPCS). SPECT results showed significant improvement after about six months of treatment for PTSD subjects. Moreover, 75\% of abnormal areas were practically indistinguishable from control subjects after the treatment. ${ }^{55}$ These data suggest that SPECT imaging can be utilized to measure an increasing number of biomolecules in vivo, in human subjects, as well as regional blood flow, and has the capacity to produce important data for PTSD and mTBI.

3.1.2. Positron emission tomography (PET). Instead of utilizing traces that directly emit $\gamma$ particles, PET tracers emit positrons through positron emission decay, which further decay into 2 gamma photons, emitted roughly $180^{\circ}$ from one another. Neural endpoints can then be visualized by means of coincidences of $\gamma$ photons hitting a detector simultaneously instead of using direct $\gamma$ emission. This has the advantage of providing more positional information than SPECT. The positrons generated by radioactive isotopes used in PET generate photons when they lose kinetic energy and interact with an electron, causing the decay of the positron into two $\gamma$ particles with the energy of $511 \mathrm{keV}$. Unlike SPECT, collimators are not required in PET because of the known directions of produced $\gamma$ photons. 
Table 2 Summary of main findings in PTSD imaging along with the contrast agents used for the study ${ }^{a}$

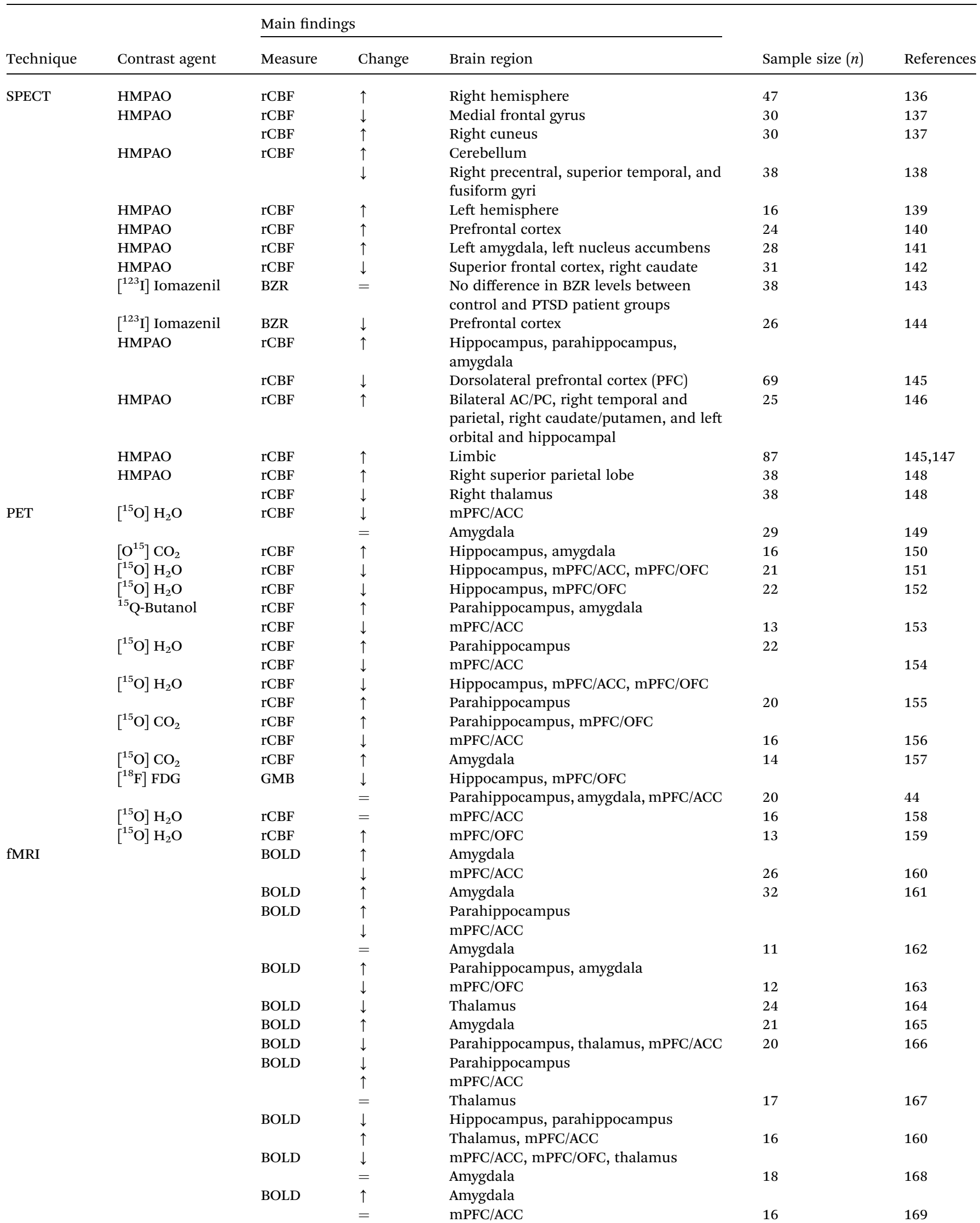


Table 2 (Contd.)

Main findings

Technique Contrast agent $\quad$ Measure Change Brain region $\quad$ Sample size $(n)$ References

${ }^{a}$ HMPAO: 99mTc hexamethyl-propyleneamine oxime; rCBF: regional cerebral blood flow; BZR: benzodiazepine receptors; AC: anterior cingulate; PC: posterior cingulate; mPFC/ACC: medial prefrontal cortex/anterior cingulated cortex; OFC: orbitofrontal cortex; FDG: fluorodeoxyglucose; GMB: glucose metabolism; BOLD: blood oxygen level-dependent signal; = : no change in activity; $\uparrow:$ increase in activity; $\downarrow$ : decrease in activity.

Brain activity and blood flow in different regions of brain can be illustrated in a 3D map by locating and measuring the amount radiotracers and utilizing mathematical approaches. Positron emitting radioactive isotopes like oxygen-15, carbon-11, fluorine-8, and nitrogen-13 are employed to label biological molecules. Generally, they are introduced into body via a gas or injection. This method is known as the most sensitive, accurate and selective neuroimaging technique due to the specificity of radiotracers and widespread variety for numerous biomolecules. Another significant advantage of this method over other types of neuroimaging techniques is the strong changes in the acquired signal $(\sim 10 \%)$ through the depth of brain that makes it a suitable technique for deep brain activity scanning. While this amount is about $1-2 \%$ for other methods like fMRI. Additionally, sensitivity loss due to the collimator is not a problem in PET, as in SPECT.

Like any neuroimaging technique, this method also has some drawbacks, including the short half-life of radiotracers such that on-site cyclotron production of radiotracers is required, making PET much more expensive. Furthermore, PET also requires specialized chemists be present to bind these radiotracers to the bioactive molecules immediately after their creation. These fiscal factors should be taken into consideration as well when comparing imaging modalities. As a result, PET is not the most accessible device for neuroimaging. PET also has other drawbacks. The temporal resolution of this method is also limited (60-100 s) in comparison with fMRI technique which is less than $2 \mathrm{~s}$. This is the main reason why fMRI and PET techniques are used in blocked and event-related designs or in tandem, in advanced PET-fMRI machines. PET also has no capacity to image targets not associated with the biomolecule employed. This makes it difficult to use PET alone when particular regional data is required. For this reason, PET often paired with computed tomography X-ray scans (CT), to provide additional structural data not available with PET alone. Most importantly, like SPECT, PET also requires the injection of radioactive materials into body which can be detrimental to the patient and combining PET with CT exacerbates this risk. ${ }^{\mathbf{1 0}, 56}$ Despite these limitations, PET is the most advanced imaging technique now available for imaging molecular factors in human patients and can be especially powerful when paired with fMRI, which does have the drawback of additional radiation exposure.

PET has already been employed in numerous studies on PTSD. A study conducted by Bremner et al. investigated the effects of mindful built stress reduction (MBSR) on combat
PTSD veterans by means of PET imaging and measurements. ${ }^{57}$ These investigators used radiolabeled heavy water to measure regional neuronal activation. Patients were randomized into groups treated with either MBSR or present centered group therapy (PCGT). Exposure to combat related sounds and pictures resulted in hyper activation of temporal cortical and frontal brain areas in all PTSD patients. On the other hand, activation of cerebellum, insula and subcortical regions dropped considerably in PTSD, compared to control patients. MBSR treated PTSD patients not only showed a greater reduction in the clinician administered PTSD scale (CAPS) than the PCGT group, but also showed an increased activation of the right anterior cingulate, right inferior parietal lobe and decreased activation of the right insula and precuneus compared to the PCGT group when exposed to trauma associated stimuli. In the previously mentioned study by Holmes et al., investigators used $\left[{ }^{18} \mathrm{~F}\right]$ FPEB, a radioligand for mGluR5 to measure this receptor by PET in human PTSD patients and observed it to be upregulated in several brain regions. Furthermore, post mortem brain samples were analyzed and an upregulation of SHANK-1 proteins which binds to mGluR5 was observed. Reduced expression of FKBP5 was also observed indicating altered glucocorticoid function in postmortem PTSD brains. ${ }^{58}$ This study exhibits the efficacy of combining molecular and imaging approaches to study particular molecular endpoints in PTSD. Additionally, in the previous study by Fabio et al. the aforementioned SPECT ligand, SRX246 is additionally useful as a radiolabeling candidate for PET imaging that can be used for PTSD related studies. ${ }^{53}$ Finally, in another study by S. E. Holmes, a PET tracer for TSPO, [ $\left.\left({ }^{11} \mathrm{C}\right)(\mathrm{R})-\mathrm{PK} 11195\right]$ a translocator protein associated with activated microglia and commonly increased by inflammation was used to show increased inflammation in the anterior cingulate cortex in depressed patients. ${ }^{59}$ These data collectively show the efficacy of PET in measuring molecular targets associated with a wide range of systems involved in PTSD, but as with TSPO, have yet to be studied in PTSD in particular.

3.1.3. Functional magnetic resonance imaging (fMRI). In this neuroimaging method, there is no need to inject radiotracers for the measurement of neural activation. In fact, the natural properties of human body are utilized as a tracer for this purpose, such as hemoglobin. In general, the existence of heme groups in red blood cells are used to create signals by means of a powerful (1.5-9 Tesla) magnet. In this approach, a magnetic field aligns protons in a specific direction. Protons are then excited by means of controlled radio frequency energy. This 
excitation causes protons to move out of alignment and then to emit radio frequency pulses as a result of relaxation back into alignment with the majority of other protons in the body. Hydrogen atoms consist of only one proton and 1 electron and compose a large portion of biological entities due to its high representation in water and lipid molecules. The majority of structural data from an MRI comes from the imaging of hydrogen atoms. fMRI is based on the imaging of hemoglobin in blood and produces a blood oxygen level dependent signal that is interpreted as an indirect signature of neural activation. The different behavior of various oxidized states of hemoglobin make it an appropriate choice as a signaling molecule in fMRI. Differential regional activation of specific brain regions can be demonstrated due to the changes in oxidized forms of hemoglobin that creates the emission of radio frequency pulses with different intensities. Absence of radiotracer materials in this technique makes it a favorable imaging method that can be easily repeated without the concern of being over-exposed to radioactive materials. fMRI is widely accessible and reasonably expensive compared to other methods, making this method a favorable approach compared to PET and SPECT when measuring brain function. Moreover, the spatial and anatomical resolutions of fMRI are remarkably higher than other common neuroimaging techniques. ${ }^{60}$

fMRI also has some important limitations. First, the temporal resolution of this device for rCBF changes is not perfect. There is a 5 second delay between neural activation and measurement, potentially causing temporal artifacts. This should be corrected in data analysis, but nonetheless adds noise to the data. Considering the high resolution of structural MRI and enhanced PET (modified with high resolution research tomography (HRRT)) which are less than $1 \mathrm{~mm}$ and $2 \mathrm{~mm}$, respectively, fMRI provides lower resolution of $3 \mathrm{~mm}$. Another serious disadvantage of fMRI is the inability of this technique to study patients with metallic implants and pacemakers, due to serious interactions of the powerful magnet with metals. The fMRI scan requires that a subject spend a significant amount of time in a relatively narrow tube in which the scan is taken, often causing anxiety and claustrophobia. The device also produces a considerable amount of noise that might either distract the patient from accomplishing a task or prohibits the investigation of medication effects by inducing anxiety in the patient, producing additional nose in the data through the activation of stress related pathways. ${ }^{\mathbf{1 0 , 6 0}}$ Finally, unlike PET and SPECT, fMRI only able to study brain function and is unable to study molecular factors associated with disease conditions.

A recent PTSD related fMRI study was carried out by Garfinkel et al. regarding the impaired contextual modulation of memories in PTSD. ${ }^{61}$ These authors observed impaired extinction recall, higher amygdala activity and increased skin conductance in PTSD subjects compared to controls. An additional emerging benefit of fMRI is the ability to provide real time neurofeedback to patients, as a therapeutic technique or to enhance experimental paradigms. Neurofeedback allows patients to use real time data from their own brains to correct disease specific differences in neural activity associated with their disorder. Zotev et al. utilized fMRI neurofeedback in real time in order to help PTSD patients modify the interactions of the amygdala and prefrontal cortices. ${ }^{62}$ It was observed that PTSD subjects experienced significantly increased blood oxygenation levels (BOLD) action in the left amygdala while accomplishing happy emotion induction tasks. After treatment, $80 \%$ of patients experiencing fMRI neurofeedback saw significant reductions in their CAPS scores, while only $33 \%$ saw reduction in their CAPS scores in the sham group. This result showed that real-time fMRI neurofeedback procedure is capable of correcting the deficiencies in the functional connectivity of amygdala and prefrontal cortex that are specific to PTSD, at least for a short period of time after treatment. Gerin et al. also took advantage of real time neurofeedback to study this novel treatment in combat veterans with PTSD. ${ }^{63}$ fMRI analysis of participants after the treatment showed high resting-state functional connection (rsFC) patterns. Patients also showed reductions in CAPS scores, further illustrating that real time neural feedback is capable of altering resting state functional connectivity in PTSD patients that provides clinically significant changes in symptoms.

3.1.4. Diffusion tensor imaging (DTI). DTI is an MRI-based technique currently utilized to study white matter tractography (WMT) in the brain. DTI takes advantage of the ability of moving water molecules to generate contrast in MRI images. This technique, first presented in $1994,{ }^{64}$ is capable of imaging myelinated connections in the brain, the major highways by which distant brain regions communicate. DTI takes advantage of the fact that water molecules diffuse through myelinated tissues in parallel substantially faster than they do perpendicular to them. ${ }^{65}$ The primary outcome measure of DTI is fractional anisotropy (FA), the difference between the parallel and perpendicular movement of water molecules in the brain. These differences are not observed in non-myelinated tissue however, with anisotropy approaching zero in grey matter and CSF. ${ }^{66}$ DTI is also rotationally invariant, allowing DTI to detect the positions of nerve fibers in space and allowing for 3D information to be generated. ${ }^{67}$ Data is often presented in a $2 \mathrm{D}$ image with a simple red-green-blue color scheme where fibers crossing from left to right are pictured in red, fibers crossing anteriorlyposteriorly in green and inferiorly to superiorly in blue, ${ }^{68}$ Virtual $3 \mathrm{D}$ trajectories of myelinated bundles can now be created using the 3D information present in the DTI analysis. ${ }^{69}$ DTI has some limitations however. There is ongoing debate over the physiological meaning of DTI images. Numerous cellular components are involved in producing WMT data including the oligodendrocytes that produce the myelin sheaths, the sheaths themselves, and the axons around which the sheaths wrap. Any of these components could play the central role in the FA measured in myelinated tissues as we don't know why myelination allows for the directional movement of water molecules in myelinated axons, but not unmyelinated ones. ${ }^{67}$

DTI has already been utilized to study mild traumatic brain injury (mTBI) ${ }^{70}$ and PTSD along with schizophrenia, ${ }^{71}$ depression $^{72}$ and neurodegenerative disorders, like Alzheimer's disease. ${ }^{73}$ The literature suggests that PTSD is associated with disrupted white matter tractography in the cingulum angular bundle (CAB) as measured by fractional anisotropy (FA). The 
CAB connects the ACC to the MTL and Hipp, possibly underlying a deficit in emotional regulation. ${ }^{74,75}$ Other investigations reported lower mean diffusivity (MD) in the $\mathrm{CAB}$, but not lower FA. ${ }^{76}$ In contrast, some reports have also exhibited elevated FA in the $\mathrm{CAB}$ and other relevant networks. ${ }^{77}$ Other studies did not observe differences in the CAB, but instead showed WMT changes in uncinate fasciculus, a white matter tract connecting the AMY to the vmPFC, a deficit possibly underlying reduced inhibitory control over the fear response. ${ }^{78}$ Other investigators have taken a networking approach and reported distinct differences in the white matter tracts of the salience network between PTSD and control participants. ${ }^{67,79}$

Overall, these studies are preliminary and few current literature reviews or metanalyses on this topic are available. Future investigations could benefit from pairing DTI investigations of WMT with fMRI data to determine the relationships between WMT and the observed functional changes, especially in the context of a network based perspective. Further investigations should also consider differences between PTSD patients and trauma exposed non-PTSD participants, which appear to be lacking in the DTI literature. Finally, future investigations in animal models and eventually humans could benefit substantially from the pairing of DTI in PTSD models with Raman spectroscopy and other molecular analyses, like lipidomics and proteomics to determine if correlations can be drawn between white matter integrity measured by DTI and a biochemical and molecular analysis of white matter tracts by the molecular techniques.

3.1.5. Magnetic resonance spectroscopy (MRS). MRS is an MRI based technique capable of non-invasively measuring differences tissue biochemical composition and holds great promise for the investigation of regional neurotransmitter differences in psychiatric disease. MRS became relevant around 1995 when the FDA approved the PROBE sequence: PROton Brain Examination, an acronym for localized, single voxel hydrogen ion (proton) spectroscopy. PROBE is rapid, automated and inexpensive for neuro-MRS. PROBE produces a proton spectrum for either a single voxel or multiple voxels as part of a protocol. PROBE can be performed on almost any MRI over $1 \mathrm{~T}$ as long as the machine can shimmer, or optimize field homogeneity. Similar to fMRI, localization of the signal can be achieved through narrowband radio frequency pulses combined with pulsed spatial gradients produced through other means. MRS can produce in vivo biochemical information in human subjects. Various biochemical materials can be identified by their spectra and although there may be some overlap at lower $\mathrm{T}$, at higher $\mathrm{T}$, this is not the case. Spectra are showed on an $X Y$ axis in which the $x$ axis plots the frequency chemical shift in parts per million of the various metabolites in a sample. The $y$ axis plots the amplitude of the signal and therefore the amount of a particular substance. The types of compounds that can be identified are partially dependent on the echo timeline (TE). For example, at $1.5 \mathrm{~T}$ and between 144 and $288 \mathrm{~ms}, \mathrm{~N}$-acetylaspartate (NAA), choline (Cho), creatine (Cr), alanine (Ala) and lactate can be measured. At $\mathrm{TE}<40 \mathrm{~ms}$, one can detect myoinositol (Myo), glutamate and glutamine (Glx), glucose (Gc) and some larger proteins and lipids. ${ }^{80}$
The literature suggests that PTSD is related with a reduction in the neuronal marker, NAA in left Hipp compared to fit control and trauma-exposed controls and in the right only against healthy controls. Cho was also shown to differ in the left hemisphere compared to healthy controls, but not trauma exposed controls. A study employing a $\mathrm{Cho} / \mathrm{Cr}$ as the measure also indicated a reduction in Cho in the ACC. One new study published in 2017 used a powerful 7 T MRS was to study 43 PTSD and mTBI patients compared to 15 controls and the investigators made the observation that NAA/Ch levels significantly decreased in parts of the left hippocampus in patients with both PTSD and mTBI compared to PTSD patients alone. ${ }^{81}$

Like DTI, the literature on MRS is small and not very many large metanalyses are available. MRS has incredible potential, but is currently limited by the very small number of compounds available for measure and the requirement of a large magnet to obtain high quality results. Alternatively, MRS is the only way in which we can currently measure any neurotransmitters directly in vivo, in human patients and its possible that future technological advances could provide a more diverse set of analytes measurable by MRS, more regional specificity, and the capacity for more granular measures.

\subsection{Pre-clinical imaging technologies}

Quantitative and objective information is significant for the improvement of disease diagnosis and treatment in PTSD. Recent advances in the utilization of novel biomedical technologies make it possible to acquire such information from biological tissues. Various optical spectroscopy methods like Fourier transform infrared (FTIR), Raman spectroscopy, near infrared spectroscopy and fluorescence spectroscopy have been employed to achieve this goal. These methods can provide vital information about the chemical composition of biological tissues at the molecular level that is strikingly beneficial for PTSD studies. Some of the most promising emergent techniques in this area are discussed in the following.

3.2.1. Fourier-transform infrared (FTIR) imaging. One of the recent fast-growing methods of studying biological tissues is Fourier transform infrared (FTIR). ${ }^{82}$ In this approach, midinfrared electromagnetic waves $\left(4000-400 \mathrm{~cm}^{-1}\right)$ are utilized to investigate the samples. The absorption of electromagnetic waves generates a unique characteristic of molecular vibrations that originate from fundamental transitions of vibrational modes. Many of the chemical species in a sample can be revealed by highly characteristic patterns with this nondestructive and label-free method. These two properties can be considered as remarkable advantages of FTIR over other emerging methods like confocal fluorescence spectroscopy that requires labeling or utilizing weak physical characteristics of materials like auto-fluorescence or refractive properties. Application of FTIR for biological samples is considerably favored due to the low energy electromagnetic waves employed in this technique. This particular feature minimizes the probability of the occurrence of any damage or photo bleaching in biological tissues or cells. During the last decade, FTIR has proven to be a powerful tool for analytical investigation of biological samples 
by means of focal or linear array detectors in a quite short time frame..$^{\mathbf{8 3 , 8 4}}$

The distribution of particular chemicals can be illustrated in label-free chemical maps via the spectral data and absorbance spectrum in the region of interest. This technique can be utilized in various applications, from static systems to dynamic ones. Tissue biopsies, ${ }^{85}$ fixed cells, ${ }^{86}$ drug diffusion,${ }^{87}$ protein crystallization, ${ }^{88}$ and live cell studies ${ }^{89}$ are some of the reported applications of the aforementioned method. The biomedical application, sample size, surrounding matrix, analyte concentration and some other variables can change in this type of imaging, relative to other techniques, allowing for different experimental designs. According to the desired application, various thermal sources as well as a synchrotron are employed to produce IR light for several FTIR imaging. Furthermore, several FTIR imaging modes such as macro imaging, micro imaging, transmission, transflection, and attenuated total reflection (ATR) methods can be utilized to accomplish imaging. Each of these modes are limited by either the image pixel size or the light diffraction. For instance, the dimension of the pixel is usually larger than the diffraction limit in macromode imaging in which pixel size is the determining factor for spatial resolution. On the other hand, diffraction of light is the significant limiting factor when the pixel dimension is smaller than the limit of diffraction. There have been several attempts to increase the spatial resolution of FTIR imaging due to the sub-micron nature of biological samples. In some cases, projected pixel size has been reported incorrectly as the limiting factor of spatial resolution. Meanwhile, projected pixel size becomes significant only in macro FTIR imaging and measurements. ${ }^{\mathbf{8 3}, 84}$

The use of a hemispherical lens above the specimen is a common method for enhancing the FTIR image quality. Initially, this method has been employed to eliminate optical aberrations caused by sandwiching the specimen between IR transparent windows in transmission FTIR imaging. Meanwhile, the numerical aperture of the system increases correspondingly by a factor of lens refractive index value due to installation of an additional hemispherical lens. ${ }^{\mathbf{8 3 4 8 , 9 0}}$ ATR imaging mode can usually generate higher spatial resolution by not transmitting IR light through the specimen. Meanwhile, the internal reflection of IR light at the interface of specimen (low refractive index) and ATR (high refractive index) occurs within the system. ${ }^{83}$

The typical method of sample preparation includes fixing the sample in a medium, optimal cutting temperature compound (OCT), and mounting the sample on a glass substrate. Fixing usually takes place by means of paraffin and the thickness of sliced samples during OCT is about $5 \mathrm{~mm}$ to $10 \mathrm{~mm}$. Due to the easy availability of sectioned samples which are already used for standard light microscopy, transmission mode is the most favored approach for FTIR imaging of tissues. ${ }^{83}$

This chemically specific, label free and non-destructive method is capable of being used in various aspects of biology, including protein detection in brain tissues. According to the literature, the chemical composition of biological samples can be investigated without the occurrence of any photo damage or disturbance to living tissues. This can be considered as a significant superiority over other methods like fluorescent labeling. However, the substantial drawbacks of FTIR imaging are low detection limit, limited spatial resolution and considerable background absorbance. These issues can be partially ameliorated by means of synchrotron sources of photons. Synchrotron sources have been proven to be a strong instrument, in order to conquer the limitations of FTIR imaging technique by increasing the signal to noise ration and spatial resolution in protein imaging. ${ }^{83,84}$

The literature reveals that FTIR has been utilized to investigate Lewy bodies (LBs) in the brain. ${ }^{91}$ The LBs are atypical deposits of alpha-synuclein protein ( $\alpha$ Syn) and its fluctuations lead to problems with cognition, movement, behavior, and mood. Lewy body dementia (LBD) is a disease most commonly linked with Alzheimer's or Parkinson's disease (PD) but can also be involved in TBI, PTSD, and Dementia. A recent research reported by Mitchell Bermans' Lab at Mississippi State suggested that the aSyn gene polymorphism controls alcohol use a common PTSD symptom..$^{92}$ Hence, the measurement of $\alpha$ Syn could be useful for understanding PTSD. The presence of high aSyn, a protein a biomarker of PD, causes harm to dopaminergic (DA) neurons. Missense mutations or gene polymorphism in $\alpha$ Syn gene can overproduce $\alpha$ Syn, causing rare inherited forms of PD. To understand $\alpha$ Syn at the cellular level, the adeno-associated virus (AAV) vector method was employed to inject foreign gene (haSyn) stereotaxically into the $\mathrm{SN}$ of mouse brain (Fig. 4(a) left). The cells were immunostained as shown in Fig. 4(b) (right). haSyn (green) and tyrosine hydroxylase (TH, red) (synthetic enzyme for DA) merged with haSyn (yellow), nucleus staining by DAPI also merged (blue). From their study, it was concluded that the DA neuronal cell death was due to phosphorylation of $\alpha$ Syn at Ser129 residue. ${ }^{93}$ Studies like this could be streamlined with novel techniques like FTIR.

In another study, synchrotron FTIR microspectroscopy was used for examination of LBs in PD subjects. This method is capable of identifying the proteins as well as investigating the changes to the secondary structure of the proteins. Fig. 4(b) (top) shows the FTIR spectra of the different sections of the protein. The spectral peaks at $\sim 1655,1630$ and $1645 \mathrm{~cm}^{-1}$ correspond to $\alpha$-helix, $\beta$-sheet, and random coil respectively. Since LBs have low density, synchrotron radiation was used to conduct this study. Shown is a FTIR image of total proteins (specifically $\beta$-sheet) of an SP (Senile plaques used for amyloid detection) in the cerebral tissue derived from patient with Alzheimer's (Fig. 4(b): left to right, bottom). Congo red stained represented the protein-rich regions. The core of the plaque was found to be rich in $\beta$-sheets and lipids. ${ }^{91}$

The information about the protein secondary structure obtained from FTIR is helpful, as they correspond to the damaged areas. Researchers have also explored FTIR spectral analysis, comparing areas of damaged and normal axons. Peaks at 1625 , $1637,1652,1664,1680$ and at $1695 \mathrm{~cm}^{-1}$ corresponded to $\beta$ sheet, random coil, ordered $\alpha$-helix, dis-ordered $\alpha$-helix, $\beta$-turn, and anti-parallel $\beta$-sheets respectively (Fig. 4(c)). The changes were identified by principal component analysis (PCA). The change in amide-II band due to $\mathrm{N}-\mathrm{H}$ and $\mathrm{C}-\mathrm{N}$ stretch was 
a
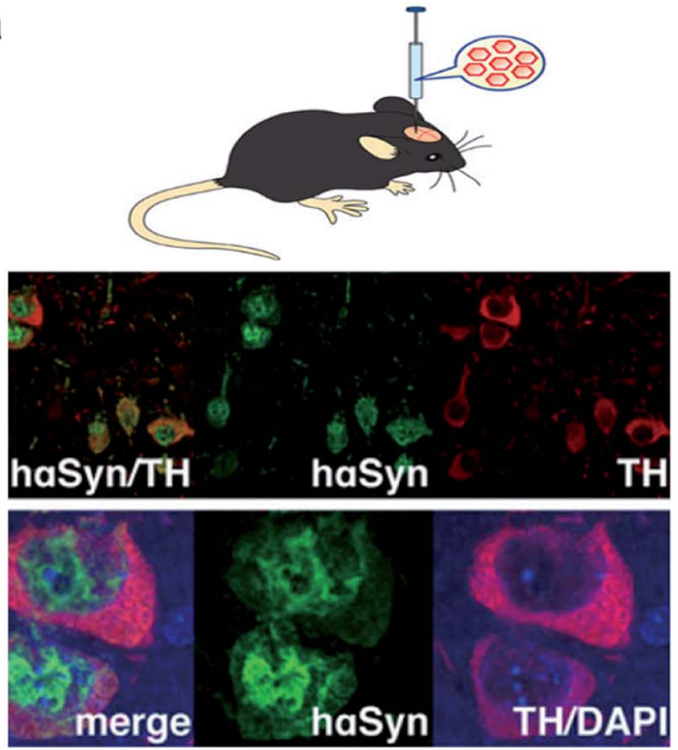

c

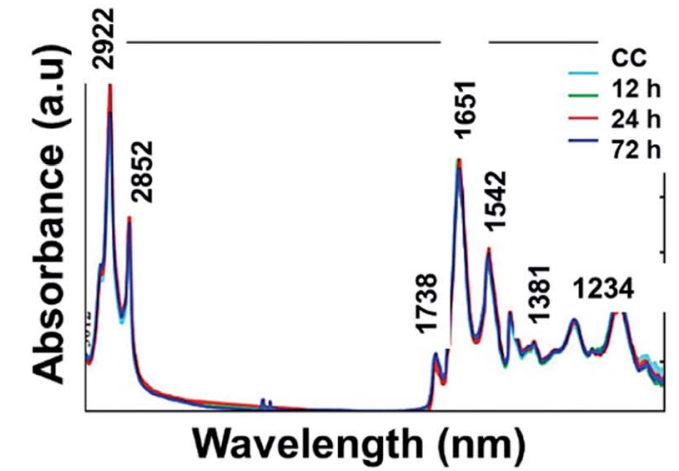

b
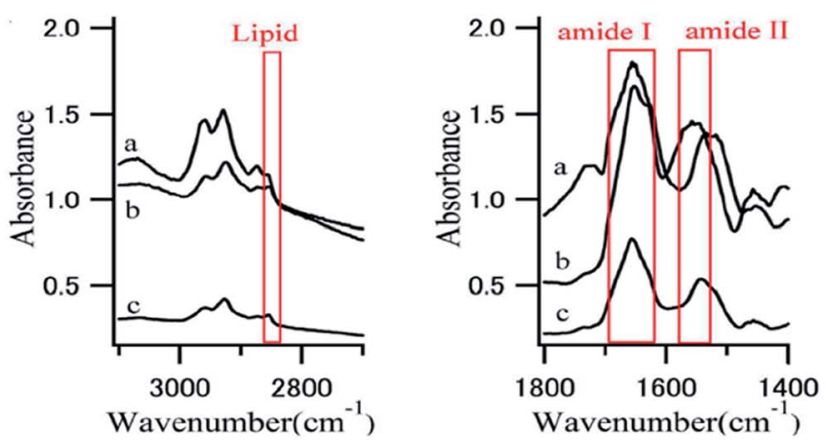

Visible Protein $\quad \beta$-sheet Lipid

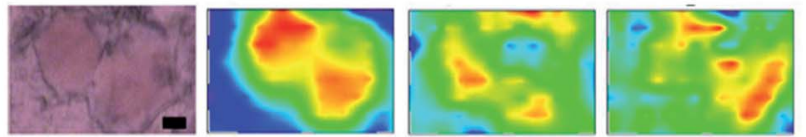

Low

High

Fig. 4 (a) AAV vector-mediated expression of foreign gene in mouse brain (left). Immunostained cells for AAV-haSyn-injected mice (right). Reproduced with permission from ref. 93. Copyright 2012, Yasuda, Nakata, and Mochizuki. (b) FTIR spectra, a: core of an LB, b: halo of an LB, and c: normal brain tissue (top). FTIR imaging of brain showing total amount of proteins (from left to right, bottom). The color bar indicates low (blue) to high (red) contents. The area in the visible image was scanned with $5 \mu \mathrm{m}$ steps $\left(16 \times 8\right.$ pixels $\left.=80 \times 40 \mu \mathrm{m}^{2}\right)$. Scale bar, $10 \mu \mathrm{m}$. Reproduced with permission from ref. 91. Copyright 2015, Macmillan Publishers Limited. (c) Secondary derivative infrared spectra of damaged and injured axons. Reproduced with permission from ref. 95. Copyright 2017, Zhang, Huang, Wang, and Dong. (d) TAl in the CC detected by b-APP staining following impact acceleration of TBI(top). A: No $\beta$-APP staining, B: $\beta$-APP-stained axons at 12 h, C: 24 h, D: 72 h (scale bar = $50 \mu \mathrm{m}$ ). Superimposition of all normalized FTIR spectra collected in the CC of rats at different post-injury groups (bottom). Reproduced with permission from ref. 94. Copyright 2015, John Wiley \& Sons.

significant in injured axons, which might be due protein conformational changes. $\beta$-APP (an amyloid precursor protein) has been known as an axonal injury biomarker and hence understanding of this using FTIR could be valuable. ${ }^{94}$

Traumatic axonal injury (TAI) was explored by another group of researchers in the corpus callosum (CC) of rats by $\beta$-APP staining post-injury at $0,12,24$, and 72 hours' time intervals, respectively. The differences were significant between the $24 \mathrm{~h}$ and other groups and were mostly from lipids. At $24 \mathrm{~h}$, there were higher intensities at 2922 and $2852 \mathrm{~cm}^{-1}$ corresponding to asymmetric and symmetric vibrations due to $\mathrm{C}-\mathrm{H}$ vibrations from $\mathrm{CH}_{2}$, and a lower asymmetric vibration at $2958 \mathrm{~cm}^{-1}$ from $\mathrm{CH}_{3}$. Lower intensities for unsaturated lipids from $\mathrm{C}=\mathrm{C}-\mathrm{CH}_{2}$ at $3012 \mathrm{~cm}^{-1}$ and band at $1738 \mathrm{~cm}^{-1}$ from lipid esters due to $\mathrm{C}=\mathrm{O}$ vibrations were also observed (Fig. 4(d) (bottom)). These findings were consistent with the histological examination as observed in Fig. 4(d) (top), wherein the biochemical changes associated with lipids and proteins become more prominent $24 \mathrm{~h}$ post injury. A disadvantage with FTIR spectroscopy is the difficulty to distinguish the different time points, depending on quantitation of the absorption peaks. Hence, statistical analysis is required to identify the subtle spectral variations. ${ }^{95}$

3.2.2. Functional near infrared spectroscopy (fNIRS). The functional near-infrared spectroscopy (fNIRS) technique has been utilized to study the cerebral injuries and disorders in human patients. ${ }^{96}$ This approach is a cost efficient, safe, noninvasive and portable technique that can be utilized for either direct or indirect monitoring. The therapeutic or optical window of light is between $650 \mathrm{~nm}$ to $950 \mathrm{~nm}$. The flux of photons inside biological samples can produce elastic or inelastic scattering, fluorescence, or absorption, but between these wavelengths, the absorption of light in water and 
hemoglobin is minimized, allowing for scattering and the detection of signal. Photons can penetrate through the tissues in the aforementioned wavelength range and detect different concentrations of hemoglobin, allowing for the derivation of a blood oxygen level dependent (BOLD) signal, much like fMRI. Scattering in tissues occurs in the forward direction and introduced by scattering coefficient $\left(\mu_{\mathrm{s}}\right)$. The scattering coefficient is defined as the inverse of average photon path length and described by the probability of scattering per unit path length in a particular medium. Photons can either be scattered by moving objects like blood cells or stagnant parts like tissues. Meanwhile, the energy of a photon is annihilated during absorption and generates luminescence or heat. The significant biological substances that absorb near infrared light are hemoglobin, water, lipids, myoglobin, cytochrome oxidase, bilirubin and melanin. The inverse average photon path length before the annihilation of a photon is defined as the absorption coefficient $\left(\mu_{\mathrm{a}}\right)$, which is approximately $0.1 \mathrm{~cm}^{-1}$ in biological tissues. The absorption properties of tissues are described by this factor. Measuring the chromophore concentrations in different wavelengths is a common method used to calculate the absorption coefficient due to the wavelength dependent nature of this factor. ${ }^{97,98}$

The most prevalent types of near infrared spectrometers are categorized as frequency domain, time domain and continuous wave NIRS. The frequency domain approach includes a head which shines with amplitude modulated NIR light. In this method, alternations of amplitude and phase are measured in the back scattered light. On the other hand, a time domain NIRS spectrometer generates short pulses of NIR and transmitted scattered photons are detected in accordance with the arrival time. However, continuous wave mode is the most ubiquitous technique in which constant amplitude and frequency are employed to illuminate the samples. In this mode, light attenuation through the sample is the only parameter that is measured. Depending on the required information, any of the aforementioned techniques can be utilized. The source of the NIR light is another significant factor that can strongly affect the final results. Generally, both light emitting diodes and fiber optic lasers are used as the source in fNIRS. However, fiber optic lasers have better maneuverability that can be applied in different sample types and positions. Required wavelength, intensity and study area are some influential factors in NIR source selection and the source detector distance (which is typically $3 \mathrm{~cm}) \cdot{ }^{97,98}$

fNIRS have been demonstrated to measure the cerebral activity in memory encoding and recovery. fNIRS utilizes in near-infrared light source to non-invasively monitor changes in the oxy (HbO-increase in blood supply) and deoxy (HbRdecrease in blood supply)-hemoglobin content in the cerebrum. $\mathrm{HbO}$ and HbR spatial and temporal concentrations are generally interrelated with the blood oxygen level dependent signals (BOLD) to track the changes in the brain. ${ }^{99}$

In another study, the temporal, parietal, and prefrontal regions were monitored to examine paired-associate learning task (PAL). ${ }^{100}$ PAL is used to evaluate memory performance/ impairment, which requires use of associative memory to pair one item to another item to which it was previously associated. A person's ability on this task depends on the individual to build a link among the paired groups. Fig. 5(a) (top) shows the designed probe used for the study and the points corresponding to the prefrontal cortex. ${ }^{\mathbf{1 0 1}}$ The results revealed that novel face encoding required activation of left inferior frontal cortex and inactivation of medial-to-superior frontal cortices which is shown to be significant from the color bar as shown in Fig. 5(a) (bottom left). These results were in accordance with fMRI and PET studies showing the left frontal gyrus activation during encoding. ${ }^{96,102}$ Fig. 5(a) (bottom right) shows that novel face recalling activated the inferior, middle, and right frontal cortices. Inactivation in middle frontal cortex through recall of the same faces might be due to the lack of attention or inactivation of a default memory network (DMN). The colored bar suggests (Fig. 5(a) bottom) participation of neural activity throughout recall, which might be due to more attentional resources. Another reason might be that harder tasks requires both high cerebral initiation and participation in comparison to easy tasks. Since these data were substantiated by other imaging techniques, fNIRS may serve as a substitute biomarker to assess encoding and retrieval memory responses in order to track a likely associated disease. In comparison to PET and fMRI, fNIRS is portable, low cost, and is non-invasive.

The phases of working memory (encoding, maintenance, and retrieval) are mediated by prefrontal activation (central executive function). A similar fNIRS study was conducted in veterans with PTSD versus healthy controls. Results obtained from statistical analysis of task-evoked hemo-dynamic responses showed that PTSD veterans had irregular prefrontal responses in comparison to healthy subjects, suggesting deficits in prefrontal control of working memory. Healthy subjects had equal activations in both hemispheres throughout all the three phases (Fig. 5(b) (middle)), while PTSD veterans exhibited initiations in the left dorsolateral prefrontal cortex (DLPFC) during encoding and maintenance (Fig. 5(b) (bottom)). In healthy controls, $\mathrm{HbO}_{2}$ had approximately $0.58 \mu \mathrm{M}$ peak (middle right) higher than the retrieval phase with approximately $0.4 \mu \mathrm{M}$ peak (middle left). For PTSD group, $\mathrm{HbO}_{2}$ changes in the forward task (bottom left) and backward task (middle right) were dominant in the maintenance phase with a discrete decline in the retrieval phase. The results were consistent with studies from Schiffer et al. ${ }^{\mathbf{1 0 3}}$ that confirmed variances in the right and left hemispheres between the healthy controls and trauma patients. The study also showed that the right frontal cortex processed negative reactions, while the left frontal cortex processed positive and neutral reactions. ${ }^{\mathbf{1 0 0}}$ Although, fNIRS is an established technique for brain imaging, most of the literature is concentrated on veterans with comorbid stage PTSD and hence the future work must be directed towards broader diagnoses covering all stages of PTSD from mild to severe, as well as PTSD derived from different types of trauma. ${ }^{\mathbf{1 0 4}}$ Recently, a handheld NIR device was attached to the scalp through optical fibers and utilized to scan brain haematomas (Fig. 5(c)). ${ }^{105}$

3.2.3. Raman spectroscopy. The basis of Raman theory is exchange of energy between light and matter. Scattering and 
a
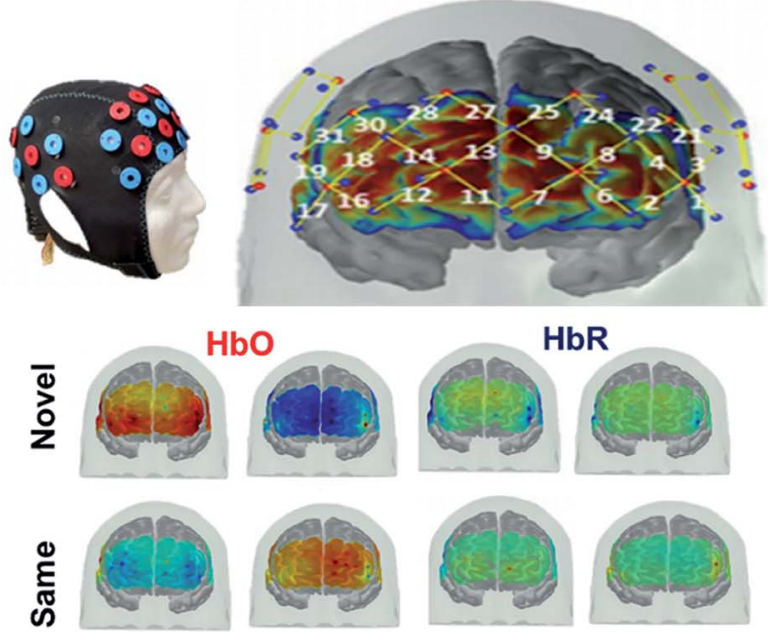

$\begin{array}{llll}0.2 & 0 & -0.2 & 0.1\end{array}$

\section{RECALL}

$\mathrm{HbO}$
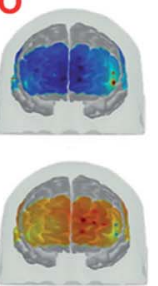

$\begin{array}{lll}0 & -0.1 & 0.1\end{array}$

ENCODE
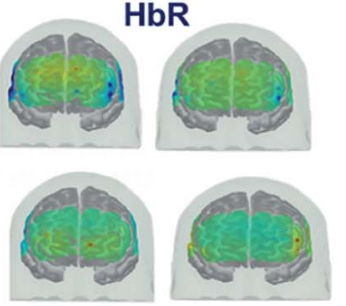

0

$\begin{array}{lll}0 & -0.1 & 0.1\end{array}$

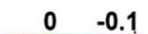

RECALL ENCODE c

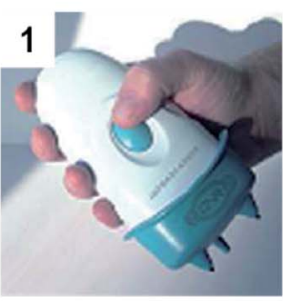

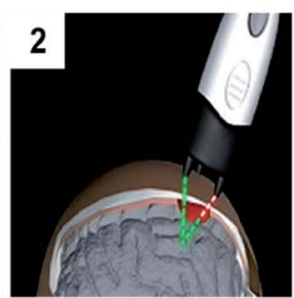

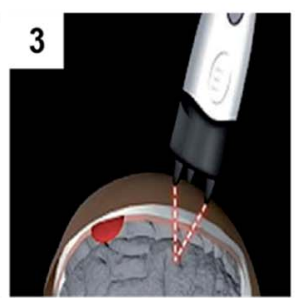

b
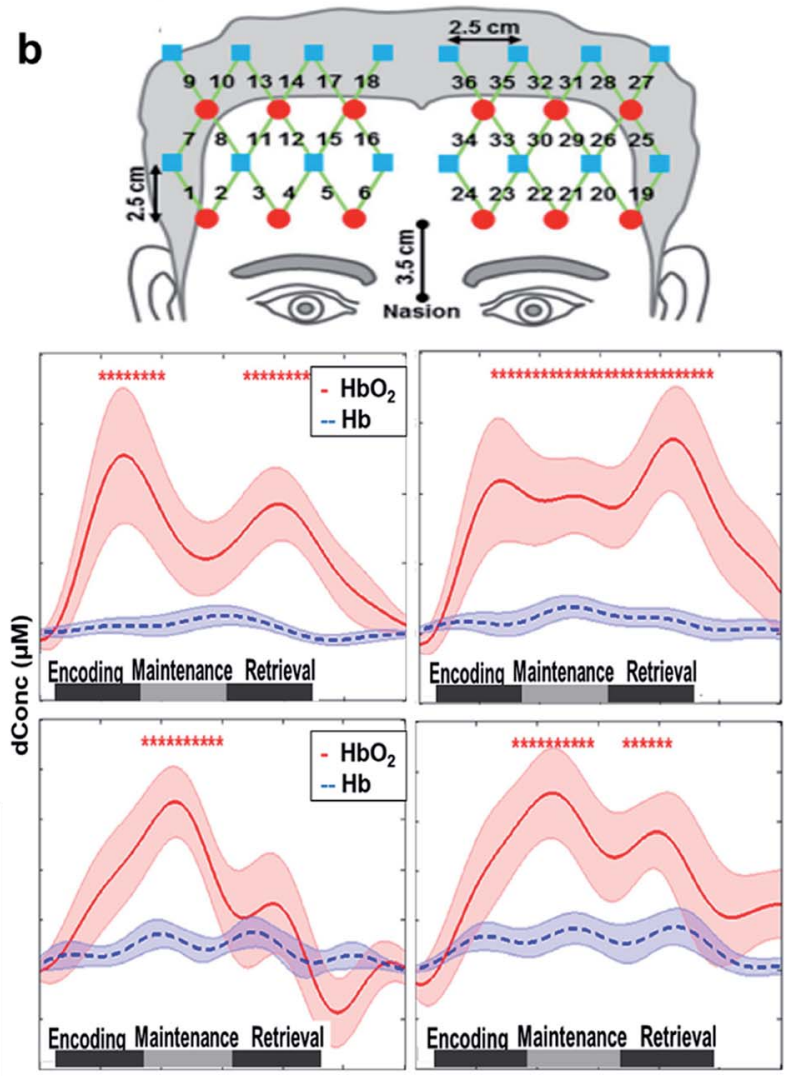

Time (s)

Fig. 5 (a) fNIRS designed probe. Location of sources (red circles) and detectors (blue circles) on the cap (top left). Sensitivity profile for the probe. The channel numbers are in yellow. The color scale spans the sensitivity logarithmically from 0.01 (blue) to 1 (red). Group mean $\mathrm{HbO}$ and $\mathrm{HbR}$ hemodynamic responses overlayed over the brain surface for: encoding (bottom left) and recalling (bottom right) of novel and same faces. The displayed concentration changed was averaged from $t=25$ to $50 \mathrm{~s}$. The color bar indicates the scale of the concentration change in $\mu \mathrm{M}$ units. Reproduced with permission from ref. 101. Copyright 2017, Nature Publishing Group. (b) Configuration (top) of the fNIRS probe. Red circles represent light sources, blue squares represent detectors, and green lines represent the nearest source-detector pairs (channels) to measure the brain activities. Mean task-evoked prefrontal hemodynamic responses in the: control group (middle) forward task (middle left) and backward task (middle right), PTSD group (bottom) forward task (bottom left) and backward task (bottom right). In both panels, the solid lines represent the mean time courses of $\mathrm{HbO}_{2}$, the dotted lines represent the mean time courses of $\mathrm{Hb}$, the shaded regions represent the standard errors, the * symbols indicate the period of significant $\mathrm{HbO}_{2}$ changes (one-sample $t$-test, $p<0.01$ ) from the baseline, and the gray bars in the bottom indicate the three phases of the task. It is noted that the actual retrieval time was slightly variable among participants. Therefore, the retrieval phases labeled in both panels are schematic and approximate. Reproduced with permission from ref. 104. Copyright 2014, Tian et al. Published by Elsevier. (c) Handheld NIR device. Bluetooth equipped Full device (part 1) intra (part 2) and extra (part 3) - axial hemorrhagic haematomas acquisition. Reproduced with permission from ref. 105. Copyright 2010, Taylor \& Francis.

absorption of light are two fundamental mechanisms in this approach. During such scattering, incident light can provoke different vibrational modes in the components of material. In this case, frequency of the incident light should be equal to the frequency of vibrational modes in the substance. In other words, Raman scattering can be considered as the absorption and emission of photons along with the molecular transition from ground state to excited state. ${ }^{106}$ This type of scattering can be measured by means of a spectrometer and quantified in the form of intensity as function of frequency. Raman peaks (bands) of different materials are unique due to the distinctive vibrational modes of molecular species. ${ }^{107,108}$

Generally, biological tissues exhibit several bands (usually $10 \mathrm{~cm}^{-1}$ to $20 \mathrm{~cm}^{-1}$ wide) in their spectra. The intensity of each peak can also demonstrate quantitative information about different chemicals in a tissue or cell. Therefore, Raman spectroscopy can be utilized as a powerful tool for either quantitative or qualitative studies of biological substances by providing distinctive fingerprints of tissues and cells. Despite the long history of Raman spectroscopy in chemical analysis of materials, the application of this technique in biology and biomedical sciences has only begun in recent years. Through technological improvements in detectors and lasers, Raman spectroscopy and microscopy has improved. Meanwhile, the current challenge in this sphere is the appropriate data analysis of Raman spectra in biological applications, which require advanced techniques of data analysis like principal component analysis (PCA). These types of analysis should focus more on information content of Raman spectra rather than only the band positions to fully take advantage of Raman data. 
Statistical, chemical, and morphological issues substantially control the data analysis quality in Raman spectroscopy and microscopy. Moreover, in terms of technological limitations, the distal tip of the probe requires more studies and improvements to be done in order to enhance the quality of data acquisition. A fully calibrated probe-tissue interface can greatly improve the acquisition of information in different depths of biological samples. Depth ranging studies of biological samples is usually limited by the natural turbidity of them caused by the elastic scattering of these media. So far, depth resolution and light collection issues have been the significant drawbacks of Raman spectroscopy and imaging in biomedical applications. These problems occur due to the weak penetration of light into samples (a few millimeters) even at low absorption conditions. At the moment, attempting to eliminate the elastic scattering and/or converting it to an advantage are being scrutinized as alternatives in designing new Raman probes. There is a great interest in the literature to provide a method in which Raman signals can penetrate much deeper into tissues to study brain injury through the skull, in PTSD patients for example. By devising a solution for probe issues in Raman microscopy, other interesting applications can also be introduced like functional imaging. Employing Raman imaging in applications such as endoscopic configurations, can generate morphological and chemical images of live tissues. For a comprehensive study of PTSD patients, Raman spectroscopy and imaging can be utilized alongside other approaches like magnetic resonance imaging, intravascular ultra sound, computed tomography scan and different types of X-ray analyses. Such studies provide complementary morphological and chemical Raman information as well as tissue density information achieved by other methods. ${ }^{107,108}$

Aforementioned methods can be used for brain imaging, but only Raman and FTIR imaging can be utilized for minimally invasive tracking of tissue biochemistry. Raman spectroscopy (RS) has also been reported to acquire label-free spectra in TBI mice. The left parietotemporal cortex of the mice brain was injured, and they were excised $7^{\text {th }}$ day post TBI, and the spectra was acquired at several spatial locations namely, contusion core (CC), contralateral (CL); pericontusional (PC), ipsilateral tissue, and distant from contusion core (ID). The spectra showed elevated levels of lipids, proteins, and cholesterol in comparison to sham control mice (Fig. 6(a) (bottom)). To locate the source of the changing lipid signal observed, RS was acquired for cholesterol, cholesteryl nonanoate, cholesteryl oleyl carbonate, intralipid, and albumin respectively. The spectra for these species in brain samples are: bands at $426,701,801$, and $1128 \mathrm{~cm}^{-1}$ corresponding to cholesterol peaks, $1301 \mathrm{~cm}^{-1}$ for fatty acids observed in cholesterol derivates, $1440 \mathrm{~cm}^{-1}$ due to $\mathrm{CH}$ stretch, $1462 \& 1660 \mathrm{~cm}^{-1}$ from $\mathrm{C}=\mathrm{C}$ stretching associated with lipids (Fig. 6(b)). Few other peaks lie within the boundary line of these peaks, which might be due to changes in protein and lipid composition post injury and trauma. Hence, RS can be used as a potential probe that is sensitive to detect pathology of TBI and can also be applied to PTSD. A disadvantage with RS is the difficulty of data interpretation and analysis to isolate the origin component, and hence requires rigorous statistical or
PCA analysis. ${ }^{109}$ Recently, handheld contact Raman spectroscopy probes have gained huge attention, and have been applied for real time acquisition in human subjects (Fig. 6(c)). ${ }^{110}$

A great strength of RS over currently available techniques is the potential to track tissue biochemistry changes in real time. Numerous studies, including recent investigations in an animal model of PTSD by Dr Francis Lab, suggest the possibility of stress induced oxidative and immune-mediated ${ }^{24}$ damage to cellular proteins, lipids, cholesterol that could be measured in real time by RS. These data could give us an idea of how quickly these changes occur after a traumatic incident and offer a measure in relation to which we could introduce therapeutics to help attenuate these stress-induced alterations to tissue biochemistry and molecular function.

3.2.4. Fluorescence microscopy. Fluorescence microscopy has been employed in many spheres of research like cell biology and neuroscience due to possessing high contrast as well as molecular specificity. Recently, fluorescence imaging techniques have flourished owing to drastic technological advances. Several parameters should be taken into consideration while choosing a desired method of fluorescence imaging depending on the required spatial resolution, sample penetration and imaging speed. Despite the huge enhancements in providing more photostable fluorophores and more advanced facilities, speed limitations of fluorescence microscopy is still a big challenge. Point scanning and parallelized systems are commonly used as two major categories of fluorescence microscopes. Laser scanning confocal microscope (LSCM) is an example of the point scanning method. In this technique, a fluorescence image is created by a single excitation focus scan of the specimen. One way to accelerate the imaging speed of the point scanning method is increasing the scan speed, which reduces the signal to noise ratio (SNR). Although the higher intensity of illumination ameliorates the aforementioned issue, photobleaching and photodamage probabilities significantly increase at higher levels of illumination intensity. Furthermore, increasing the illumination intensity higher than certain values might be ineffective due to the excitation of all existing fluorophores depending on the type of fluorophores in the sample. On the other hand, the parallelizing excitation method is usually employed when high SNR as well as high scan speed are desired. Accordingly, multiple excitation foci are utilized together to achieve this goal while illuminating the sample. To achieve the highest speed of image acquisition, the whole specimen is illuminated simultaneously, which is called widefield microscopy. Although the scan rate speed is increased dramatically by this method, contamination of in-focus light with scattered light as well as optical sectioning degradation can occur. Point scanners are usually used when higher performance is preferred to higher speed. Therefore, either point scanner systems or parallelized designs are employed depending on the desired application. For instance, deep imaging of biological tissues is generally accomplished by means of multiphoton excitation point scanner designs. Besides, in many applications like live functional imaging, scanning the whole specimen is not required and randomaccess scanning can be done by point scanners in specific 
a
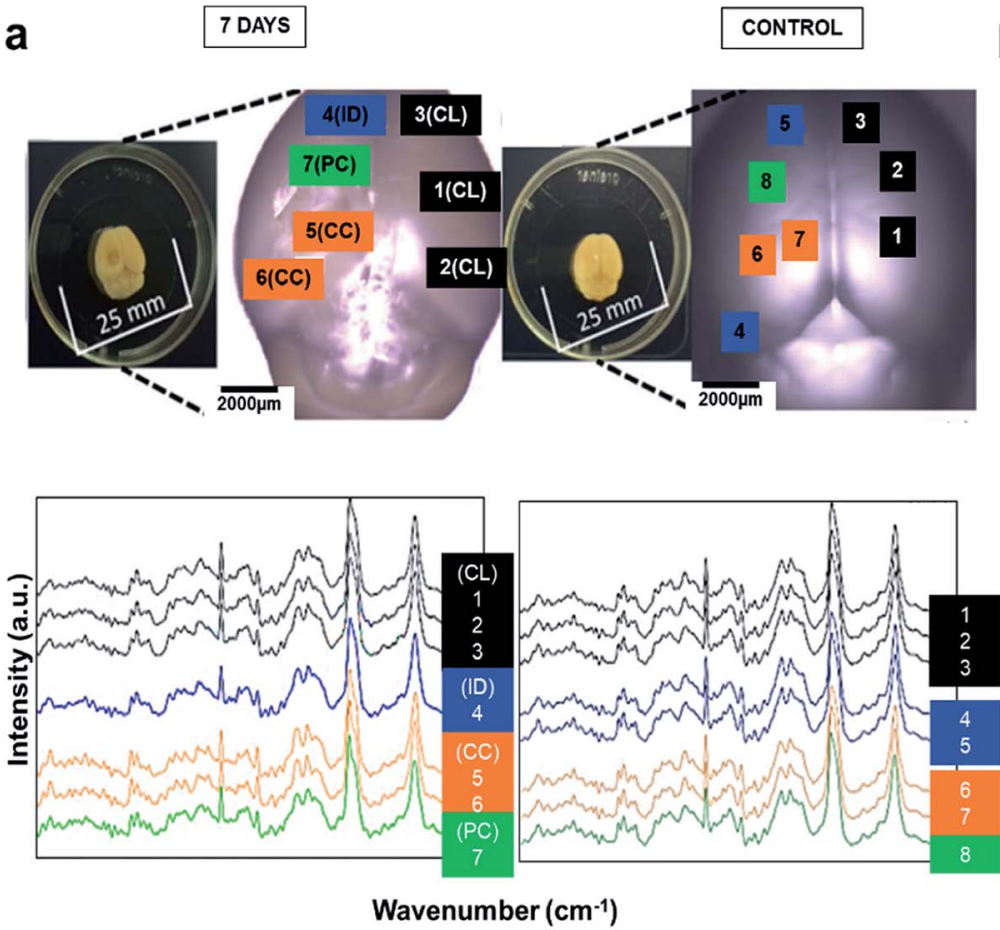

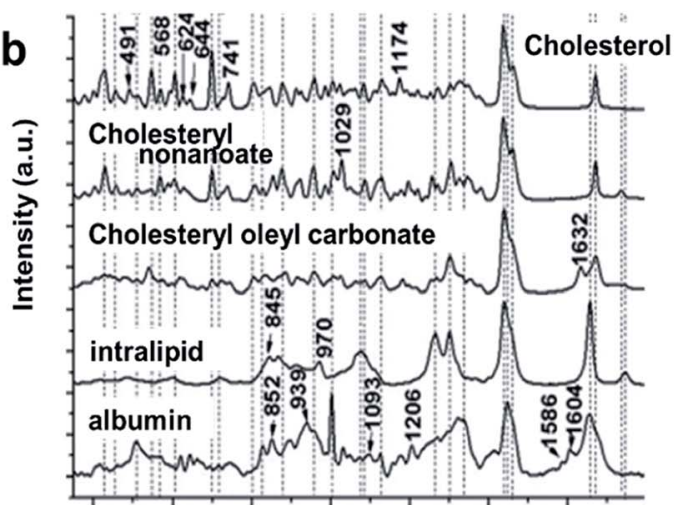

C

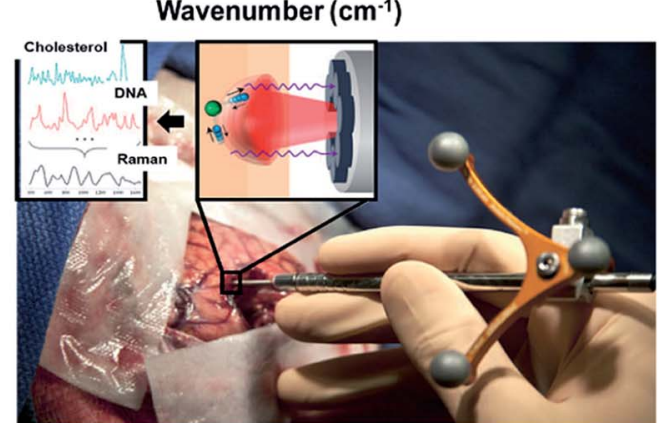

Fig. 6 Raman spectroscopy to detect tissue biochemistry. (a) Bright field microscopy of murine brains at 7th day and control (inset photographs show sample preparation). Average Raman spectra shown were acquired from the areas indicated in matching colors in the photos. $\mathrm{CL}=$ contralateral; ID = ipsilateral distant from contusion core; PC = pericontusional tissue; and CC = contusion core. Spectra were acquired at $785 \mathrm{~nm}$, with $1 \mathrm{~s}$ exposure and 10 accumulations; the average is performed over 30 separate spatial points (bottom). (b) Raman spectral of the cholesterol, cholesteryl nonanoate, cholesteryl oleyl carbonate, and intralipid (no structure was shown as mixed lipids). Protein albumin was shown for reference. Spectra were acquired at $785 \mathrm{~nm}$, with $1 \mathrm{~s}$ exposure and 10 accumulations at 175 mw; the average is performed over 10 separate spatial points. Reproduced with permission from ref. 109. Copyright 2017, Published by The Royal Society of Chemistry. (c) Image of a handheld contact Raman spectroscopy probe. Reproduced with permission from ref. 110. Copyright 2015, American Association for the Advancement of Science.

regions of interest. Light sheet fluorescence microscopy (LSFM) is another option that provides higher scan rates and SNR compared to point scanners due to the use of parallelized excitation. This method has been proven to be extremely beneficial in neurobiological and PTSD studies. LSFM method have been utilized for individual neuron studies at $0.5 \mathrm{~Hz}$ volumetric imaging rates for exposure times of more than eight hours successfully. Joint deconvolution in registering two perpendicular samples have been carried out along with rapid capturing imaging techniques to improve the temporal and spatial resolution of LSFM in some reports. This technique has been applied to obtain the images from the larvae of the zebrafish with a cellular temporal resolution of $800 \mathrm{~ms}$ per volume. ${ }^{111,112}$

In fluorescence microscopy, lateral and axial resolution are generally confined to $250 \mathrm{~nm}$ and $500 \mathrm{~nm}$, respectively. However, some super resolution devices provide higher spatial resolution than the typical diffraction limits. According to Nyquist condition, a $N$-fold resolution rise in D-dimension needs a $N \times D$-fold reduction in the magnitude of pixels. Therefore, performing super high resolution imaging requires almost eight times higher signal collection rate to maintain the same SNR of conventional devices. ${ }^{111,112}$
Multicolor fluorescence imaging provides an advantage of monitoring multiple sections after tagging it with specific fluorophores. Multi-imaging was demonstrated in a cryoinjured mice dosed with both PSS-794 (for detection of apoptotic cells) and Tracer-653 (deep-red fluorescent vascular imaging agent) for optical imaging of blood brain barrier (BBB). A 60 s cryobrain injury was delivered to 3-cohorts of hairless mice followed by single dose injection of PSS-794 at 0,3 and $7^{\text {th }}$ day respectively. Fig. 7(a) (left) shows pictures obtained after fluorescence staining using PSS-794 (apoptotic cells), Tracer-794 (vasculature), and Annexin-Vivo 750 (cellular protein for early stages of apoptosis) in a cryoinjured mouse model. Tracer-794 are seen after probe injection at the site of cryolesion but it disappears later. Extensive accumulation of Annexin-Vivo 750 was observed in the kidneys. After $5 \mathrm{~h}$, the mice were also injected with Tracer-653 and after $1 \mathrm{~h}$, they were sedated, sacrificed, and the brain were removed and positioned on an epifluorescence station for ex vivo imaging. The PSS-794 images in Fig. 7(a) (left) reveals that PSS-794 accumulation decreased with the age of the injury and the amount of cell death decreased due to removal by the mice immune system after Day 3 , as indicated by the MPI (mean pixel intensities) for a cryolesion section, which was 6-fold lower on Day 3 than on Day 0. In contrast, Tracer-653 pictures showed that BBB repair slowed 

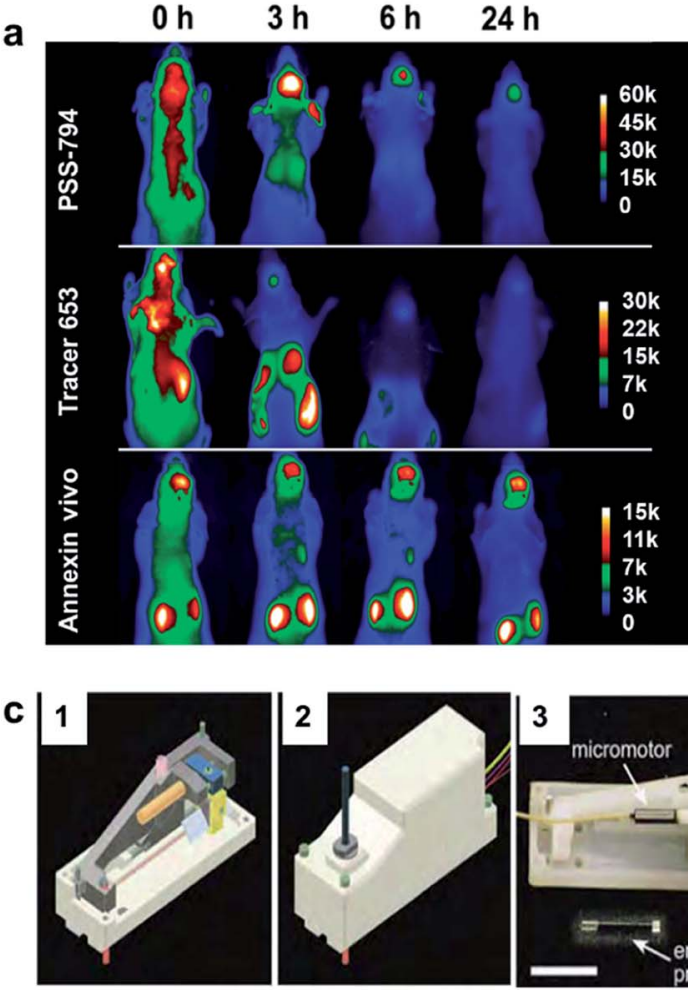
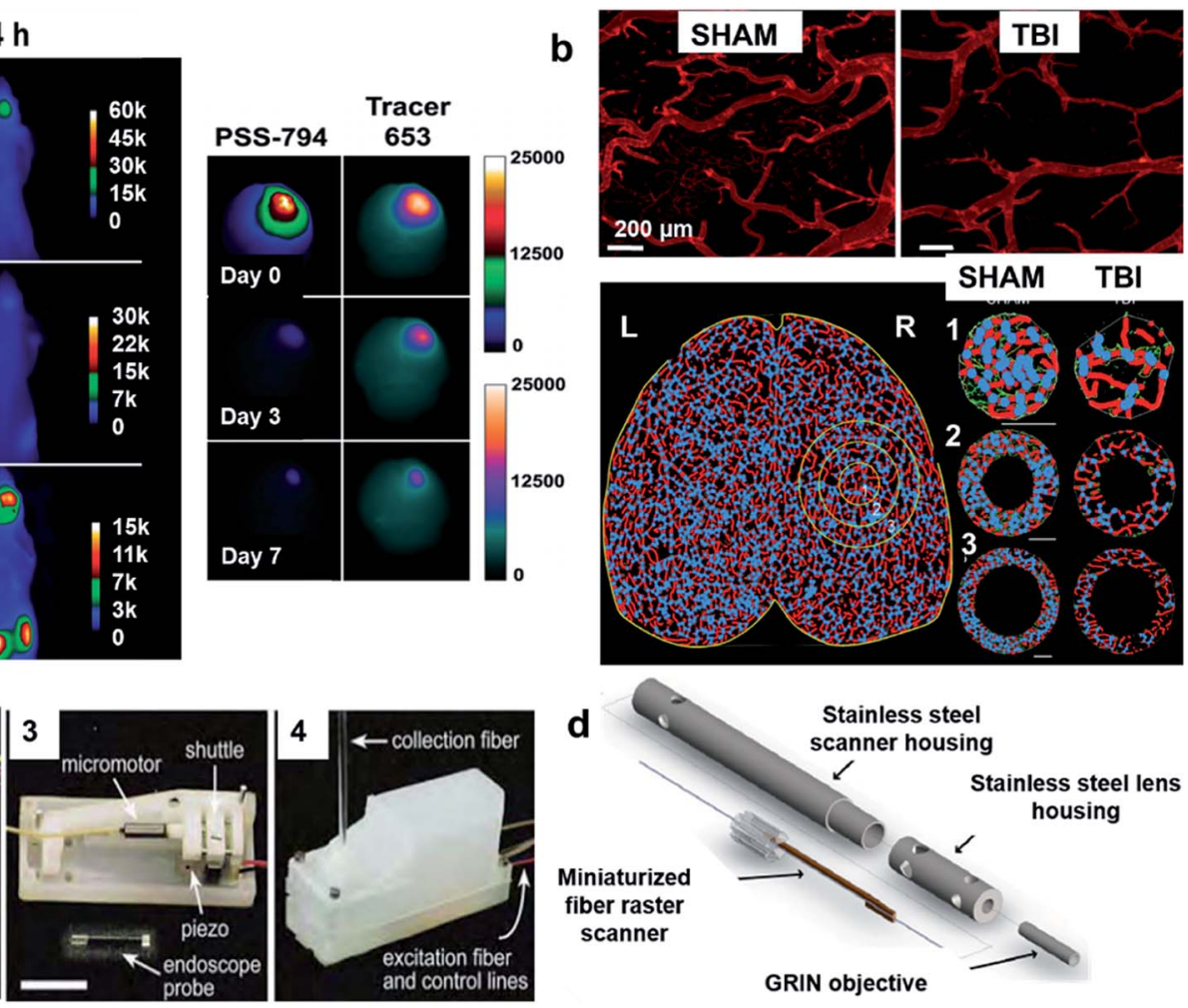

SHAM TBI
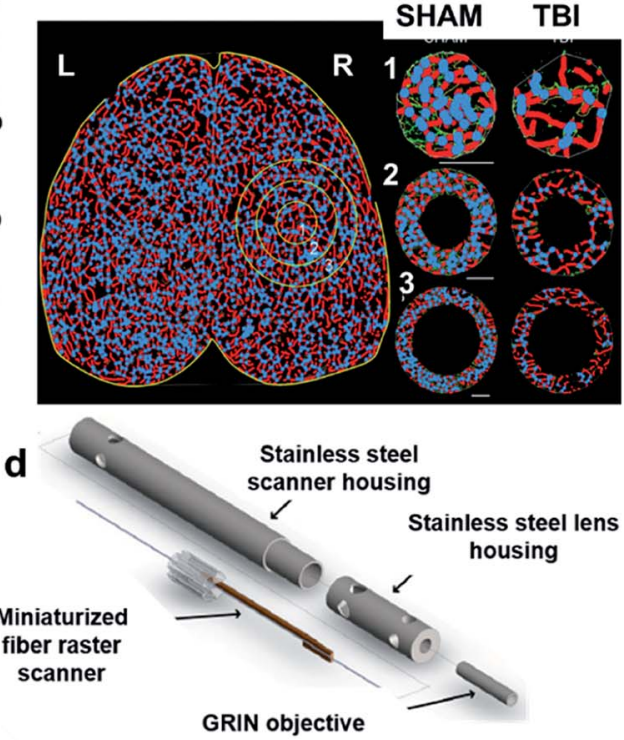

Fig. 7 (a) Representative in vivo near-infrared fluorescence assortments of PSS-794, Tracer-794, and Annexin-Vivo 750 accumulations in a brain cryoinjury mouse model. A precooled metal cylinder was applied to the head of each mouse for $60 \mathrm{~s}$ followed by intravenous injection of either PSS794 (3.0 mg kg$\left.{ }^{-1}\right)$, Tracer-794 (3.0 mg kg $\left.{ }^{-1}\right)$, or Annexin-Vivo 750. Images were acquired at the indicated time points after probe injection. $N$ $=5$. (left) Multicolor fluorescence ex vivo imaging of cell death and blood brain-barrier disruption in cryoinjured brains (right). Reproduced with permission from ref. 113. Copyright 2012, American Chemical Society. (b) Confocal images of vessel painted vasculature at the perilesional region for Sham and TBI. Cal bar $=200 \mu \mathrm{m}$ (top). Classical vascular analysis using AngioTool imaging software. Axial vessels are displayed (red) and junctions (blue). Reproduced with permission from ref. 114. Copyright 2017, Nature Publishing Group. (c) Two-photon Fluorescence Micro endoscope. Computer aided design (CAD) (part 1), top view of entire setup (part 2), internal components (part 3), mechanism of light collection (part 4). Reproduced with permission from ref. 115. Copyright 2005, The Optical Society. (d) Image of a 3 mm outer diameter multiphoton endoscope. Reproduced with permission from ref. 116. Copyright 2011, National Academy of Sciences.

with the age of injury. The BBB was porous after Day 7 than before the injury. ${ }^{113}$ In another similar study, TBI versus control results were acquired from confocal imaging of the brain vasculature. Fig. 7(b) (top) exhibited substantial damage of vessels at the perilesional region in TBI in comparison to sham control mice. Apart from the cortical imaging, several injured vascular region of interest were also measured (Fig. 7(b) (bottom)). The results showed a decrease in vessel number and length in TBI mice. From quantitative analysis, it was found that the vessel number decreased by $34.4 \%$ in TBI mice in comparison to sham controls. ${ }^{114}$ These data show how brain injury and BBB permeability can be imaged in models of TBI. Since PTSD exhibits similar characteristics to TBI, these methods could also improve the study of PTSD in animal models. Recently, a portable and light weight fluorescence endoscope was assembled for imaging both cells and tissues. Fig. 7(c), shows the computer aided design (CAD) (part 1) of the two-photon micro-endoscope, with the essential components such as the endoscope probe (part 3), piezo actuator, the excitation and the collection fiber (part 4). This device weighed $\sim 3.9 \mathrm{~g}$ making it applicable for visualization of blood vessels in the hippocampus of live mice. ${ }^{115}$ Recently, a $3 \mathrm{~mm}$ outer diameter multiphoton raster scanning endoscope was demonstrated for imagining unstained tissues (Fig. 7(d)). ${ }^{116}$

\subsubsection{Other optical imaging techniques}

Photoacoustic imaging (PAT). Photoacoustic imaging (PAT) is another emerging technique which has been demonstrated in literature recently. The acoustic/sound waves are generated from specimens underneath the skin by exposing to either radio frequency waves or by absorption of electromagnetic waves. Fig. 8(a) fig.9 (part 1) shows the schematics of PAT working along with images of the mouse brain vasculature (part 2) and the cortical vasculature of part 2 with scalp removed (part 3 ). Although PAT is promising, it is limited due to the low penetration depth resulting in low resolution, and hence is less applicable. ${ }^{117}$ Laser speckle contrast imaging (LSCI) is another emerging technique that have been utilized for acquiring the spatiotemporal dynamics of a vasculature. It is a simple technique based on the tissue contrast from dynamic scattered light. Fig. 8(b) shows the integration of the lenses for LSCI imaging. The advantage of this technique is that due to its simple configuration, it can be utilized for the cortical imaging 
a

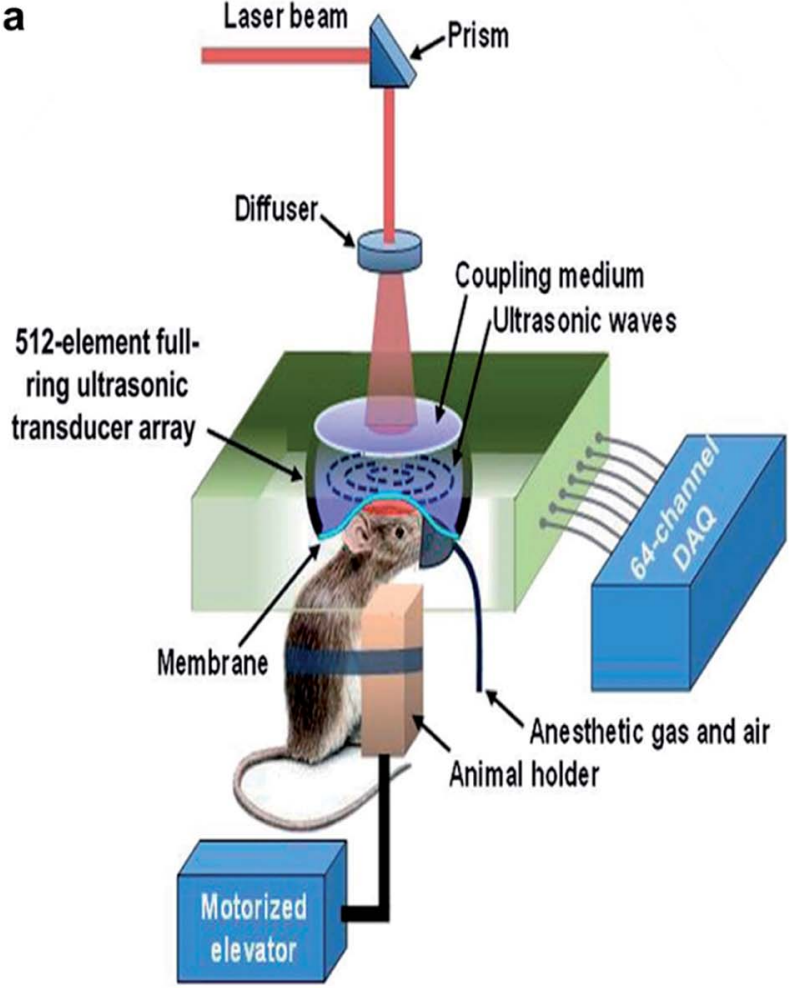

b
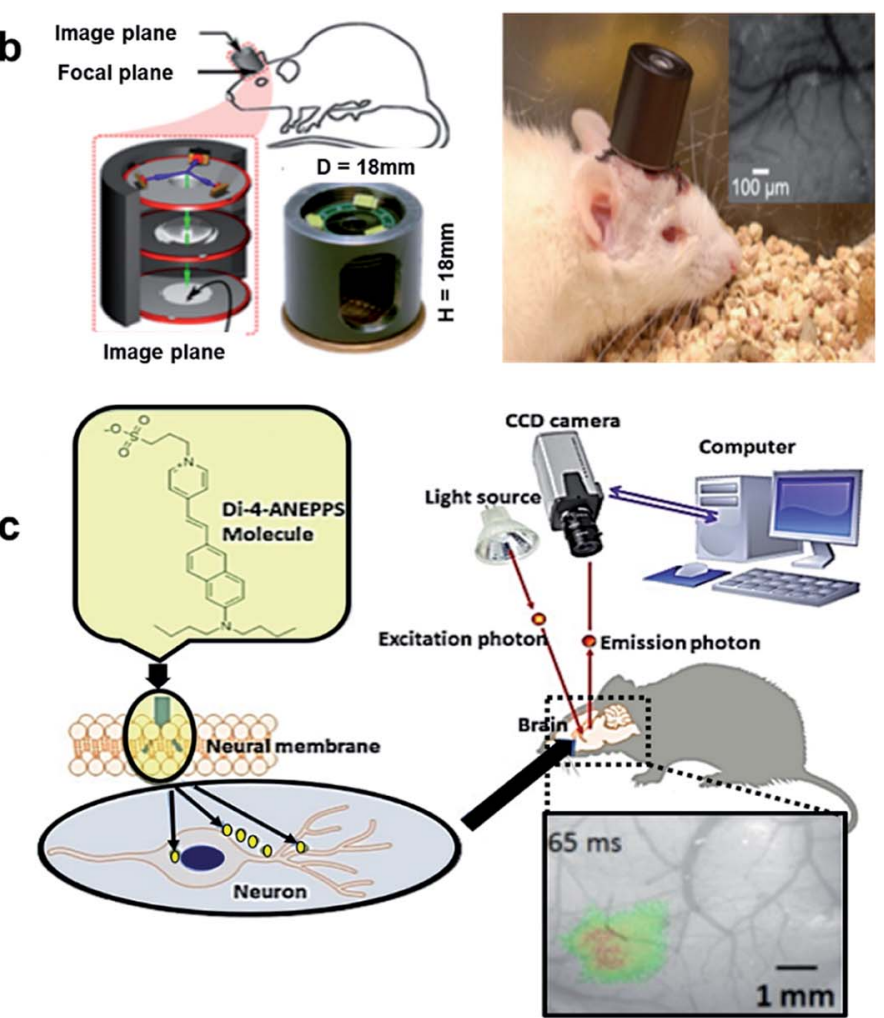

Fig. 8 Other optical imaging techniques. (a) Schematics of Photoacoustic Tomography (PAT). Reproduced with permission from ref. 117. Copyright 2014, National Academy of Sciences. (b) Schematics of the assembled Laser speckle contrast imaging (LSCl) with microscope showing incident and reflected light paths in blue and green (left), and imaging of free moving mice (right). Reproduced with permission from ref. 118. Copyright 2009, Elsevier B.V. (c) Demonstration of voltage sensitive dye imaging (VSDi) system. Schematics shows localization of dye in the membranes and a VSDi optical image acquired from the assembled setup. Reproduced with permission from ref. 119. Copyright 2013, Elsevier B.V.

of a moving mouse without tethering it as shown in Fig. 8(b) (right). This technique is limited due to its temporal resolution. ${ }^{118}$ Voltage-sensitive dye imaging (VSDi) is another technique that have been reported for visualizing neural networks with a high temporal resolution ( $\sim 1 \mathrm{~ms})$. Fig. 8(c) shows the schematics of the VSDi working. It is largely dependent on the ratio of absorbance and fluorescence w.r.t the applied membrane potential. This technique is limited by applied voltage. ${ }^{119}$

Super resolution imaging. In order to overcome the diffraction limit of light microscopy methods, various novel techniques have been introduced to enhance the resolution of microscopes. Although electron microscopy also provides a nanometric resolution for biological imaging, lack of large volume imaging, labeling of multi-targets and the need for fixation of samples are serious challenges that restrain its applications. ${ }^{120}$ As a result, more advanced light microscopy methods such as stimulated emission depletion (STED), stochastic optical reconstruction microscopy (STORM) (also known as photo-activated localization microscopy (PALM)) and structured illumination microscopy (SIM) have attracted so much interest in neurobiological field. Lateral and axial resolution of normal light microscopes are limited to 200 and $600 \mathrm{~nm}$, respectively. ${ }^{121}$ However, super resolution techniques overcome this limitation by utilizing near and far field mechanisms of electromagnetic fields. ${ }^{122}$ In STED method, the spatial resolution in the focal plane is enhanced by using a quenching laser. Although an extremely high spatial resolution of $6 \mathrm{~nm}$ has been reported in the literature, ${ }^{123}$ the achievable resolution with this method is commonly between 30-70 nm. ${ }^{124}$ The capability of this method to obtain images from live cell sub-cellular structures is the main reason of its popularity in the field of neurobiology. Thick tissue imaging is another interesting feature of STED which has been utilized for dendritic spines morphological dynamics studies of brain samples. ${ }^{125}$ Furthermore, combination of STED with neurobiological techniques has revealed strong connections between electrical properties of synapses and morphological dynamics of dendritic spines. ${ }^{126}$ On the other hand, STORM technique utilizes super resolved imaging of each fluorochrome molecule to overcome the diffraction limit of light. By this method, nanometric resolution can be achieved via calculation of the center of mass for individual fluorochromes. ${ }^{127}$ However, the fluorochromes should be activated sparsely in order to prevent overlapping. Furthermore, this approach heavily depends on image processing/analysis techniques and temporal resolution is also limited due to the huge number of acquired images $(\sim 1000)$ which are needed to be taken to form the final highresolution image. Because of the wide field nature of STORM, it is rarely used for thick tissue slices. Instead, it is usually employed for membrane protein and intracellular studies. ${ }^{128}$ In SIM, heterodyne detection method is used to continuously excite the sample in a wide field mode. In this imaging 


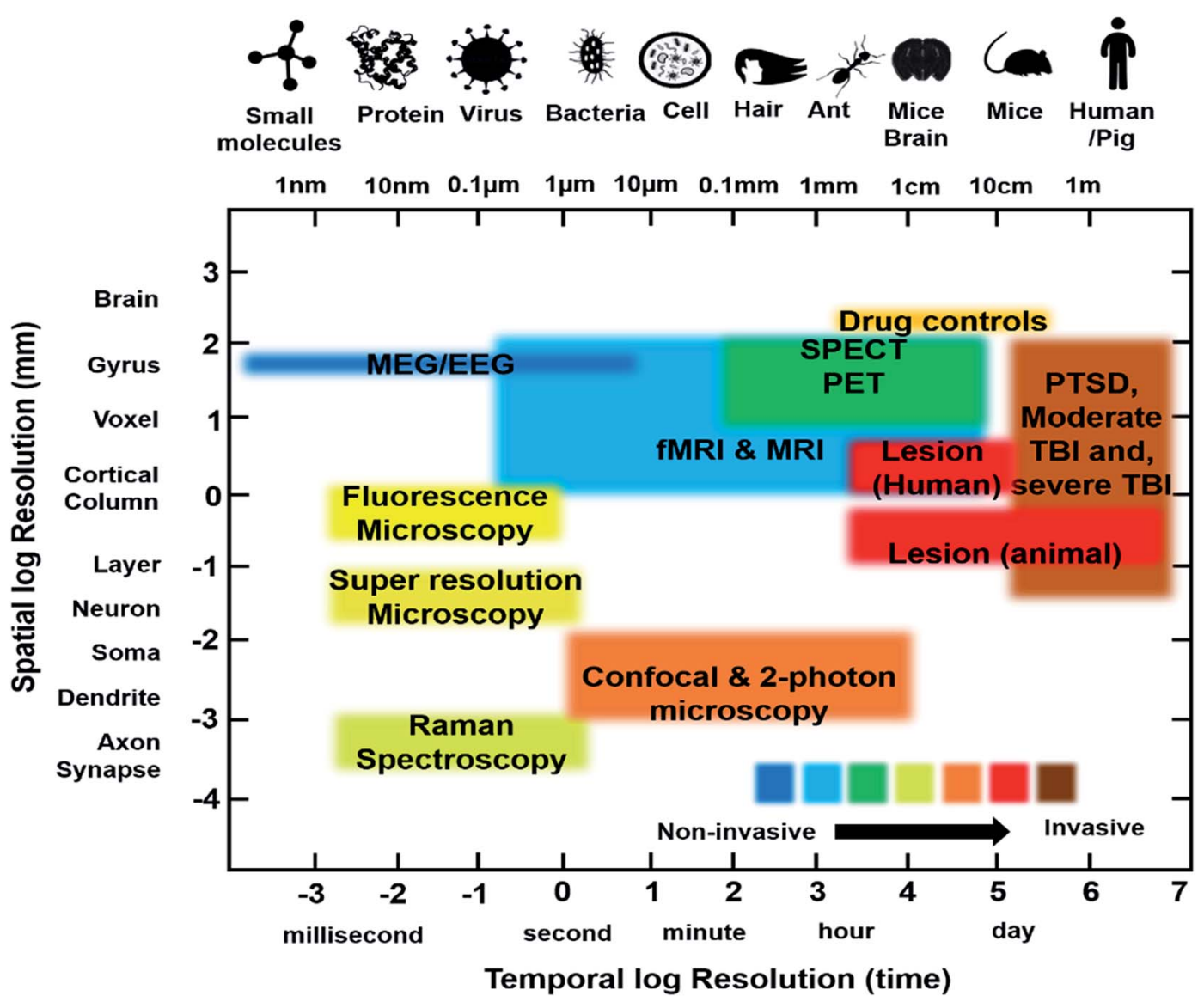

Fig. 9 Comparison of temporal and spatial resolution for conventional and emerging methods.

technique, higher spatial frequency signal is converted into a detectable bandwidth to form a fluorescence image. Although the contrast is usually low for thicker samples in this method, it provides a fast imaging $(\sim 10 \mathrm{~Hz})$ rate due to the use of wide field imaging mode. ${ }^{129}$

$4 D$ imaging. Another novel imaging technique in neurobiology studies uses ultra sound scanning to generate a $4 \mathrm{D}$ image of brain. The differences in reflection of ultra sound from various regions of brain produces contrast to form an image. Wave-return time and the strength of the returned wave constructs the image. While 3D imaging provides structural information from various parts of the brain, $4 \mathrm{D}$ imaging also generates live streaming of the region of interest. Therefore, 4D imaging is actually similar to $3 \mathrm{D}$ imaging plus providing live streaming capabilities. ${ }^{130}$ Despite the advantages of this method for live studies of brain activities, its application is mainly limited due to the low resolution $(\sim 100 \mu \mathrm{m})$ of this method compared to other emerging techniques. ${ }^{131}$ The equipment used for $4 \mathrm{D}$ ultrasound imaging is also extremely costly which makes it an unaffordable option for many cases. As a result, very few studies are available which has been utilized this approach. In a recent study, 4D ultrasound microvascular imaging was performed to visualize the brain hemodynamics without usage of contrast agents. ${ }^{132}$ They have showed the successful employment of this technique for whole brain imaging that can be used in various brain conditions and disorders. Other $4 \mathrm{D}$ imaging techniques were also rarely used for brain-related studies like 4D-CT angiography (CTA), ${ }^{133} 4 \mathrm{D}$ electrical capacitance volume tomography (ECVT) $)^{134}$ and $4 \mathrm{D}$ two-photon inverted selective plane illumination microscopy (2PE-iSPIM). ${ }^{135}$

\section{Conclusion \& future perspectives}

This review summarized the literature regarding conventional and emerging imaging and spectroscopy techniques in PTSD related studies. Fig. 9 illustrates the spatial and temporal resolution of the methods discussed in this article at a glance. Information from animal models of the disease as well as some of the most important potential biomarkers being studied were also included for a deeper comprehension of this topic and to provide example endpoints that may be important for future imaging studies. Conventional methods of investigating PSTD like SPECT, PET and fMRI were discussed along with their advantages and limitations to provide a basis for our current capacity to study PTSD. Furthermore, novel approaches for detection of PTSD biomarkers and their recent improvements and drawbacks were mentioned to provide an overview of the current literature and to create a guideline for future improvements. Conventional methods of PTSD studies are not able to detect a unique biomarker for this disease, or any psychiatric disease for that matter. All of the revealed cellular and behavioral indicators for PSTD are common to other disorders and 
this makes the diagnosis and treatment dramatically difficult. On the other hand, novel approaches like FTIR and Raman spectroscopy may be capable of introducing unique identifiers of this disease. However, some enhancements need to be made in the design of current devices to make them suitable in order to reach the aforementioned goal. Finally, emerging methods should be employed as complementary tools for conventional approaches to achieve the best results in the shortest timeline. Ultimately, a true biomarker for PTSD may be a composite formed from the analysis of several biomarkers in tandem, within the context of computational models that integrate functional and molecular imaging as well as genetic and peripheral markers of the disease. This may be the only way to develop a truly diagnostic and predictive capacity of the sort required for a comprehensive diagnosis and assessment of PTSD that could support adequate treatment plans for treatment resistant patients.

\section{Conflicts of interest}

There are no conflicts of interest to declare.

\section{Acknowledgements}

M. R. G. acknowledges the support from LSU start-up fund, Louisiana Board of Regents Support Fund (RCS Award Contract Number: LEQSF (2017-20)-RD-A-04), and LaSPACE (LEQSF (2015-18)-LASPACE, GR-4216). A. C. was supported by Economic Development Assistantship (EDA) grant. A. P. was supported by the National Science Foundation (NSF Award Number: 1660233).

\section{References}

1 C. Benjet, E. Bromet, E. Karam, R. Kessler, K. McLaughlin, A. Ruscio, V. Shahly, D. Stein, M. Petukhova and E. Hill, Psychol. Med., 2016, 46, 327-343.

2 R. C. Kessler, A. Sonnega, E. Bromet, M. Hughes and C. B. Nelson, Arch. Gen. Psychiatry, 1995, 52, 1048-1060.

3 N. Breslau and G. C. Davis, Am. J. Psychiatry, 1992, 149, 671. 4 P. N. Santiago, R. J. Ursano, C. L. Gray, R. S. Pynoos, D. Spiegel, R. Lewis-Fernandez, M. J. Friedman and C. S. Fullerton, PLoS One, 2013, 8, e59236.

5 P. R. Zoladz and D. M. Diamond, Neurosci. Biobehav. Rev., 2013, 37, 860-895.

6 American Psychiatric Association, Diagnostic and statistical manual of mental disorders (DSM-5®), American Psychiatric Pub, 2013.

7 P. J. Ebenezer, C. B. Wilson, L. D. Wilson, A. R. Nair and J. Francis, PLoS One, 2016, 11, e0160923.

8 N. Gilpin and J. J. G. Weiner, Brain Behav., 2017, 16, 15-43. 9 V. Michopoulos, A. Powers, C. F. Gillespie, K. J. Ressler and T. Jovanovic, Neuropsychopharmacology, 2017, 42, 254.

10 V. Francati, E. Vermetten and J. Bremner, Depression Anxiety, 2007, 24, 202-218.

11 U. Schmidt, S. F. Kaltwasser and C. T. Wotjak, Dis. Markers, 2013, 35, 43-54.
12 H. J. Kang, S. Yoon and I. K. Lyoo, Exp. Neurobiol., 2015, 24, 186-196.

13 L. Zhang, H. Li and R. J. Ursano, in Heat shock proteins and whole body physiology, Springer, 2010, pp. 179-192.

14 R. Yehuda, N. P. Daskalakis, F. Desarnaud, I. Makotkine, A. Lehrner, E. Koch, J. D. Flory, J. D. Buxbaum, M. J. Meaney and L. M. Bierer, Front. Psychiatry, 2013, 4, 118.

15 R. D. Marshall, K. L. Beebe, M. Oldham and R. Zaninelli, Am. J. Psychiatry, 2001, 158, 1982-1988.

16 J. G. Hensler, in Handbook of Behavioral Neuroscience, Elsevier, 2010, vol. 21, pp. 367-378.

17 C. A. Lowry and M. W. Hale, in Handbook of Behavioral Neuroscience, Elsevier, 2010, vol. 21, pp. 379-397.

18 A. C. Linthorst and J. M. Reul, in Handbook of behavioral neuroscience, Elsevier, 2010, vol. 21, pp. 475-491.

19 C. B. Wilson, P. J. Ebenezer, L. D. McLaughlin and J. Francis, PLoS One, 2014, 9, e89104.

20 M. J. Girgenti and R. S. Duman, Biol. Psychiatry, 2017.

21 E. R. De Kloet, M. Joëls and F. Holsboer, Nat. Rev. Neurosci., 2005, 6, 463.

22 D. A. Young, S. S. Inslicht, T. J. Metzler, T. C. Neylan and J. A. Ross, Psychiatry Res., 2018, 270, 961-966.

23 F. J. Raabe and D. Spengler, Front. Psychiatry, 2013, 4, 80.

24 C. B. Wilson, L. D. McLaughlin, A. Nair, P. J. Ebenezer, R. Dange and J. Francis, PLoS One, 2013, 8, e76146.

25 K. Speer, D. Upton, S. Semple and A. McKune, J. Inflammation Res., 2018, 11, 111.

26 R. Yehuda, L. C. Pratchett, M. W. Elmes, A. Lehrner, N. P. Daskalakis, E. Koch, I. Makotkine, J. D. Flory and L. M. Bierer, Interface Focus, 2014, 4, 20140048.

27 C. Anacker, P. A. Zunszain, L. A. Carvalho and C. M. Pariante, Psychoneuroendocrinology, 2011, 36, 415425.

28 M.-L. Meewisse, J. B. Reitsma, G.-J. De Vries, B. P. Gersons and M. Olff, Br. J. Psychiatry, 2007, 191, 387-392.

29 C. Anacker, P. A. Zunszain, A. Cattaneo, L. A. Carvalho, M. J. Garabedian, S. Thuret, J. Price and C. M. Pariante, Mol. Psychiatry, 2011, 16, 738.

30 C. Anacker, A. Cattaneo, K. Musaelyan, P. A. Zunszain, M. Horowitz, R. Molteni, A. Luoni, F. Calabrese, K. Tansey and M. Gennarelli, Proc. Natl. Acad. Sci. U. S. A., 2013, 110, 8708-8713.

31 D. Mehta, T. Klengel, K. N. Conneely, A. K. Smith, A. Altmann, T. W. Pace, M. Rex-Haffner, A. Loeschner, M. Gonik and K. B. Mercer, Proc. Natl. Acad. Sci. U. S. A., 2013, 110, 8302-8307.

32 A. Karl, M. Schaefer, L. S. Malta, D. Dörfel, N. Rohleder and A. Werner, Neurosci. Biobehav. Rev., 2006, 30, 1004-1031.

33 D. C. O'Doherty, K. M. Chitty, S. Saddiqui, M. R. Bennett and J. Lagopoulos, Psychiatry Res., Neuroimaging, 2015, 232, 1-33.

34 S. H. Woodward, D. G. Kaloupek, C. C. Streeter, C. Martinez, M. Schaer and S. Eliez, Biol. Psychiatry, 2006, 59, 582-587.

35 T. J. Akiki, C. L. Averill and C. G. Abdallah, Curr. Psychiatry Rep., 2017, 19, 81. 
36 R. Yehuda, J. A. Golier, L. Tischler, P. D. Harvey, R. Newmark, R. K. Yang and M. S. Buchsbaum, J. Psychiatr. Res., 2007, 41, 435-445.

37 V. Van Veen and C. S. Carter, Physiol. Behav., 2002, 77, 477482.

38 K. Izuma, M. Matsumoto, K. Murayama, K. Samejima, N. Sadato and K. Matsumoto, Proc. Natl. Acad. Sci. U. S. A., 2010, 107, 22014-22019.

39 S. L. Rauch, L. M. Shin, E. Segal, R. K. Pitman, M. A. Carson, K. McMullin, P. J. Whalen and N. Makris, NeuroReport, 2003, 14, 913-916.

40 K. S. LaBar, J. C. Gatenby, J. C. Gore, J. E. LeDoux and E. A. Phelps, Neuron, 1998, 20, 937-945.

41 J. E. Dunsmoor, S. E. Prince, V. P. Murty, P. A. Kragel and K. S. LaBar, NeuroImage, 2011, 55, 1878-1888.

42 R. J. Dolan, Philos. Trans. R. Soc., B, 2007, 362, 787-799.

43 T. V. Gurvits, M. E. Shenton, H. Hokama, H. Ohta, N. B. Lasko, M. W. Gilbertson, S. P. Orr, R. Kikinis, F. A. Jolesz and R. W. McCarley, Biol. Psychiatry, 1996, 40, 1091-1099.

44 J. D. Bremner, R. B. Innis, C. K. Ng, L. H. Staib, R. M. Salomon, R. A. Bronen, J. Duncan, S. M. Southwick, J. H. Krystal and D. Rich, Arch. Gen. Psychiatry, 1997, 54, 246-254.

45 K. Kasai, H. Yamasue, M. W. Gilbertson, M. E. Shenton, S. L. Rauch and R. K. Pitman, Biol. Psychiatry, 2008, 63, 550-556.

46 P. R. Szeszko and R. Yehuda, Psychiatry Res., 2019, 277, 5257.

47 P. L. S. Jacques, P. A. Kragel and D. C. Rubin, Cogn. Affect. Behav. Neurosci., 2013, 13, 554-566.

48 M. Tursich, T. Ros, P. Frewen, R. Kluetsch, V. D. Calhoun and R. A. Lanius, Acta Psychiatr. Scand., 2015, 132, 29-38.

49 P. V. Bayly, E. H. Clayton and G. M. Genin, Annu. Rev. Biomed. Eng., 2012, 14, 369-396.

50 P. Garrigue, L. Giacomino, C. Bucci, V. Muzio, M. A. Filannino, F. Sabatier, F. Dignat-George, P. Pisano and B. Guillet, Int. J. Stroke, 2016, 11, 117-126.

51 M. T. Asl, R. Nemati, F. Yousefi, H. Salimipour, I. Nabipour and M. Assadi, Iran. J. Nucl. Med., 2017, 25, 1-14.

52 C. A. Raji and T. A. Henderson, Neuroimaging Clin., 2018, 28, 67-82.

53 K. Fabio, C. Guillon, C. J. Lacey, S.-f. Lu, N. D. Heindel, C. F. Ferris, M. Placzek, G. Jones, M. J. Brownstein and N. G. Simon, Bioorg. Med. Chem., 2012, 20, 1337-1345.

54 M. Q. Hoexter, G. Fadel, A. C. Felício, M. B. Calzavara, I. R. Batista, M. A. Reis, M. C. Shih, R. K. Pitman, S. B. Andreoli and M. F. Mello, Psychopharmacology, 2012, 224, 337-345.

55 P. G. Harch, S. R. Andrews, E. F. Fogarty, J. Lucarini and K. W. Van Meter, Med. Gas Res., 2017, 7, 156.

56 C. Wang, M. S. Placzek, G. C. Van de Bittner, F. A. Schroeder and J. M. Hooker, ACS Chem. Neurosci., 2016, 7, 484-489.

57 J. D. Bremner, S. Mishra, C. Campanella, M. Shah, N. Kasher, S. Evans, N. Fani, A. J. Shah, C. Reiff, L. L. Davis, V. Vaccarino and J. Carmody, Front. Mol. Psychiatry, 2017, 8, 157.
58 S. E. Holmes, M. J. Girgenti, M. T. Davis, R. H. Pietrzak, N. DellaGioia, N. Nabulsi, D. Matuskey, S. Southwick, R. S. Duman and R. E. Carson, Proc. Natl. Acad. Sci. U. S. A., 2017, 114, 8390-8395.

59 S. E. Holmes, R. Hinz, S. Conen, C. J. Gregory, J. C. Matthews, J. M. Anton-Rodriguez, A. Gerhard and P. S. Talbot, Biol. Psychiatry, 2018, 83, 61-69.

60 A. R. Mayer, P. S. Bellgowan and F. M. Hanlon, Neurosci. Biobehav. Rev., 2015, 49, 8-18.

61 S. N. Garfinkel, J. L. Abelson, A. P. King, R. K. Sripada, X. Wang, L. M. Gaines and I. Liberzon, J. Neurosci., 2014, 34, 13435-13443.

62 V. Zotev, R. Phillips, M. Misaki, C. K. Wong, B. E. Wurfel, F. Krueger, M. Feldner and J. Bodurka, NeuroImage Clin., 2018, 19, 106-121.

63 M. I. Gerin, H. Fichtenholtz, A. Roy, C. J. Walsh, J. H. Krystal, S. Southwick and M. Hampson, Front. Mol. Psychiatry, 2016, 7, 111.

64 P. J. Basser, J. Mattiello and D. LeBihan, Biophys. J., 1994, 66, 259-267.

65 P. J. Basser and C. Pierpaoli, Magn. Reson. Med., 1998, 39, 928-934.

66 C. Pierpaoli, P. Jezzard, P. J. Basser, A. Barnett and G. Di Chiro, Radiology, 1996, 201, 637-648.

67 Y. Assaf and O. Pasternak, J. Mol. Neurosci., 2008, 34, 51-61.

68 S. Pajevic and C. Pierpaoli, Magn. Reson. Med., 1999, 42, 526-540.

69 M. Catani, R. J. Howard, S. Pajevic and D. K. Jones, NeuroImage, 2002, 17, 77-94.

70 A. Klimova, M. S. Korgaonkar, T. Whitford and R. A. Bryant, Biol. Psychiatry Cogn. Neurosci. Neuroimaging, 2019, 4, 8190.

71 M. Kubicki, R. McCarley, C.-F. Westin, H.-J. Park, S. Maier, R. Kikinis, F. A. Jolesz and M. E. Shenton, J. Psychiatr. Res., 2007, 41, 15-30.

72 Y. Liao, X. Huang, Q. Wu, C. Yang, W. Kuang, M. Du, S. Lui, Q. Yue, R. C. Chan and G. J. Kemp, J. Psychiatry Neurosci., 2013, 38, 49.

73 A. R. Hoy, M. Ly, C. M. Carlsson, O. C. Okonkwo, H. Zetterberg, K. Blennow, M. A. Sager, S. Asthana, S. C. Johnson and A. L. Alexander, PLoS One, 2017, 12, e0173982.

74 C. L. Averill, L. A. Averill, K. M. Wrocklage, J. C. Scott, T. J. Akiki, B. Schweinsburg, S. M. Southwick, J. H. Krystal and C. G. Abdallah, Mol. Neuropsychiatry, 2018, 4, 75-82.

75 N. Fani, T. Z. King, T. Jovanovic, E. M. Glover, B. Bradley, K. Choi, T. Ely, D. A. Gutman and K. J. Ressler, Neuropsychopharmacology, 2012, 37, 2740.

76 L. M. Bierer, I. Ivanov, D. M. Carpenter, E. W. Wong, J. A. Golier, C. Y. Tang and R. Yehuda, Psychoneuroendocrinology, 2015, 51, 567-576.

77 K. Aschbacher, S. H. Mellon, O. M. Wolkowitz, C. HennHaase, R. Yehuda, J. D. Flory, L. M. Bierer, D. Abu-Amara, C. R. Marmar and S. G. Mueller, Brain Imaging Behav., 2018, 12, 989-999. 
78 S. B. Koch, M. Van Zuiden, L. Nawijn, J. L. Frijling, D. J. Veltman and M. Olff, J. Psychiatry Neurosci., 2017, 42, 331.

79 Z. Long, X. Duan, B. Xie, H. Du, R. Li, Q. Xu, L. Wei, S.-x. Zhang, Y. Wu and Q. Gao, J. Affective Disord., 2013, 150, 798-806.

80 D. Soares and M. Law, Clin. Radiol., 2009, 64, 12-21.

81 A. Karl and A. Werner, Neurosci. Biobehav. Rev., 2010, 34, 722.

82 Z. Movasaghi, S. Rehman and D. I. Ur Rehman, Appl. Spectrosc. Rev., 2008, 43, 134-179.

83 K. A. Chan and S. G. Kazarian, Chem. Soc. Rev., 2016, 45, 1850-1864.

84 A. C. S. Talari, M. A. G. Martinez, Z. Movasaghi, S. Rehman and I. U. Rehman, Appl. Spectrosc. Rev., 2017, 52, 456-506.

85 S. M. Levine and D. L. B. Wetzel, Appl. Spectrosc. Rev., 1993, 28, 385-412.

86 P. Bassan, H. J. Byrne, J. Lee, F. Bonnier, C. Clarke, P. Dumas, E. Gazi, M. D. Brown, N. W. Clarke and P. Gardner, Analyst, 2009, 134, 1171-1175.

87 M. Hartmann, B. D. Hanh, H. Podhaisky, J. Wensch, J. Bodzenta, S. Wartewig and R. H. Neubert, Analyst, 2004, 129, 902-905.

88 M. J. Hackett, J. Lee, F. El-Assaad, J. A. McQuillan, E. A. Carter, G. E. Grau, N. H. Hunt and P. A. Lay, ACS Chem. Neurosci., 2012, 3, 1017-1024.

89 R. Zhao, L. Quaroni and A. Casson, Analyst, 2010, 135, 5361.

90 M. J. Baker, J. Trevisan, P. Bassan, R. Bhargava, H. J. Butler, K. M. Dorling, P. R. Fielden, S. W. Fogarty, N. J. Fullwood and K. A. Heys, Nat. Protoc., 2014, 9, 1771-1791.

91 K. Araki, N. Yagi, Y. Ikemoto, H. Yagi, C.-J. Choong, H. Hayakawa, G. Beck, H. Sumi, H. Fujimura and T. Moriwaki, Sci. Rep., 2015, 5, 17625.

92 C. R. Guillot, J. R. Fanning, T. Liang, A. M. Leventhal and M. E. Berman, J Anxiety Disord., 2015, 30, 41-47.

93 T. Yasuda, Y. Nakata and H. Mochizuki, Mol. Neurobiol., 2013, 47, 466-483.

94 J. Zhang, F. Niu, H. Dong, L. Liu, J. Li and S. Li, J. Forensic Sci., 2015, 60, 759-763.

95 J. Zhang, P. Huang, Z. Wang and H. Dong, Biosci. Rep., 2017, 37, BSR20170720.

96 S. Wijeakumar, T. J. Huppert, V. A. Magnotta, A. T. Buss and J. P. Spencer, NeuroImage, 2017, 147, 204-218.

97 D. R. Patel, Investigation of Prefrontal Cognition Responses to Transcranial Laser Stimulation in PTSD Using Functional Near Infrared Spectroscopy, MS thesis, University of Texas at Arlington, 2016.

98 M. A. Yücel, J. J. Selb, T. J. Huppert, M. A. Franceschini and D. A. Boas, Curr. Opin. Biomed. Eng., 2017, 4, 78-86.

99 T. J. Huppert, R. D. Hoge, S. G. Diamond, M. A. Franceschini and D. A. Boas, NeuroImage, 2006, 29, 368-382.

100 F. Schiffer, M. H. Teicher, C. Anderson, A. Tomoda, A. Polcari, C. P. Navalta and S. L. Andersen, Behav. Brain Funct., 2007, 3, 13.
101 S. Jahani, A. L. Fantana, D. Harper, J. M. Ellison, D. A. Boas, B. P. Forester and M. A. Yücel, Sci. Rep., 2017, 7, 9533.

102 M. K. Sidhu, J. Stretton, G. P. Winston, S. Bonelli, M. Centeno, C. Vollmar, M. Symms, P. J. Thompson, M. J. Koepp and J. S. Duncan, Brain, 2013, 136, 1868-1888.

103 F. Schiffer, M. H. Teicher and A. C. Papanicolaou, J. Neuropsychiatry Clin. Neurosci., 1995, 7(2), 169-175.

104 F. Tian, A. Yennu, A. Smith-Osborne, F. Gonzalez-Lima, C. S. North and H. Liu, NeuroImage Clin., 2014, 4, 808-819.

105 J. Leon-Carrion, J. M. Dominguez-Roldan, U. LeonDominguez and F. Murillo-Cabezas, Brain Inj., 2010, 24, 1193-1201.

106 A. Chaichi, A. Prasad and M. Gartia, Biosensors, 2018, 8, 107.

107 E. Hanlon, R. Manoharan, T. W. Koo, K. Shafer, J. Motz, M. Fitzmaurice, J. Kramer, I. Itzkan, R. Dasari and M. Feld, Phys. Med. Biol., 2000, 45, R1.

108 D. Fu, Curr. Opin. Chem. Biol., 2017, 39, 24-31.

109 J. M. Surmacki, L. Ansel-Bollepalli, F. Pischiutta, E. R. Zanier, A. Ercole and S. E. Bohndiek, Analyst, 2017, 142, 132-139.

110 M. Jermyn, K. Mok, J. Mercier, J. Desroches, J. Pichette, K. Saint-Arnaud, L. Bernstein, M.-C. Guiot, K. Petrecca and F. Leblond, Sci. Transl. Med., 2015, 7, 274 ra219.

111 P. W. Winter and H. Shroff, Curr. Opin. Chem. Biol., 2014, 20, 46-53.

112 R. Yuste, Nat. Methods, 2005, 2, 902-904.

113 B. A. Smith, B.-W. Xie, E. R. van Beek, I. Que, V. Blankevoort, S. Xiao, E. L. Cole, M. Hoehn, E. L. Kaijzel and C. W. Löwik, ACS Chem. Neurosci., 2012, 3, 530-537.

114 A. Obenaus, M. Ng, A. M. Orantes, E. Kinney-Lang, F. Rashid, M. Hamer, R. A. DeFazio, J. Tang, J. H. Zhang and W. J. Pearce, Sci. Rep., 2017, 7, 239.

115 B. A. Flusberg, J. C. Jung, E. D. Cocker, E. P. Anderson and M. J. Schnitzer, Opt. Lett., 2005, 30, 2272-2274.

116 D. R. Rivera, C. M. Brown, D. G. Ouzounov, I. Pavlova, D. Kobat, W. W. Webb and C. Xu, Proc. Natl. Acad. Sci. U. S. A., 2011, 108(43), 17598-17603.

117 M. Nasiriavanaki, J. Xia, H. Wan, A. Q. Bauer, J. P. Culver and L. V. Wang, Proc. Natl. Acad. Sci. U. S. A., 2014, 111, 21-26.

118 K. Murari, E. Greenwald, R. Etienne-Cummings, G. Cauwenberghs and N. Thakor, Annual International Conference of the IEEE Engineering in Medicine and Biology Society, Minneapolis, September, 2009.

119 L.-D. Liao, V. Tsytsarev, I. Delgado-Martínez, M.-L. Li, R. Erzurumlu, A. Vipin, J. Orellana, Y.-R. Lin, H.-Y. Lai and Y.-Y. Chen, Biomed. Eng. Online, 2013, 12, 38.

120 R. Chéreau, J. Tønnesen and U. V. Nägerl, Methods, 2015, 88, 57-66.

121 A. N. Boettiger, B. Bintu, J. R. Moffitt, S. Wang, B. J. Beliveau, G. Fudenberg, M. Imakaev, L. A. Mirny, C.-t. Wu and X. Zhuang, Nature, 2016, 529, 418.

122 F. Huang, G. Sirinakis, E. S. Allgeyer, L. K. Schroeder, W. C. Duim, E. B. Kromann, T. Phan, F. E. Rivera-Molina, J. R. Myers and I. Irnov, Cell, 2016, 166, 1028-1040. 
123 E. Rittweger, K. Y. Han, S. E. Irvine, C. Eggeling and S. W. Hell, Nat. Photonics, 2009, 3, 144.

124 J. Tønnesen, V. K. Inavalli and U. V. Nägerl, Cell, 2018, 172, 1108-1121.

125 U. V. Nägerl, K. I. Willig, B. Hein, S. W. Hell and T. Bonhoeffer, Proc. Natl. Acad. Sci. U. S. A., 2008, 105, 18982-18987.

126 K. Takasaki and B. L. Sabatini, Front. Neuroanat., 2014, 8, 29.

127 A. Hainsworth, S. Lee, P. Foot, A. Patel, W. Poon and A. Knight, Neuropathol. Appl. Neurobiol., 2018, 44, 417-426.

128 J. Tønnesen and U. V. Nägerl, Exp. Neurol., 2013, 242, 3340.

129 L. Schermelleh, P. M. Carlton, S. Haase, L. Shao, L. Winoto, P. Kner, B. Burke, M. C. Cardoso, D. A. Agard and M. G. Gustafsson, Science, 2008, 320, 1332-1336.

130 D. Smythe, 3D/4D ultrasound imaging system, US Pat. 6,702,745 B1, 2004.

131 J. Sauvage, J. Porée, C. Rabut, G. Férin, M. Flesch, B. Rosinski, A. Nguyen-Dinh, M. Tanter, M. Pernot and T. Deffieux, IEEE International Ultrasonics Symposium (IUS), Kobe, Japan, October, 2018.

132 C. Demené, E. Tiran, L.-A. Sieu, A. Bergel, J. L. Gennisson, M. Pernot, T. Deffieux, I. Cohen and M. Tanter, NeuroImage, 2016, 127, 472-483.

133 P. W. Willems, P. Taeshineetanakul, B. Schenk, P. A. Brouwer, K. G. Terbrugge and T. Krings, Neuroradiology, 2012, 54, 123-131.

134 W. P. Taruno, M. R. Baidillah, R. I. Sulaiman, M. F. Ihsan, S. E. Fatmi, A. H. Muhtadi, F. Haryanto and M. Aljohani, IEEE 10th International Symposium on Biomedical Imaging, San Francisco, USA, April, 2013.

135 Z. Lavagnino, G. Sancataldo, M. d'Amora, P. Follert, D. D. P. Tonelli, A. Diaspro and F. C. Zanacchi, Sci. Rep., 2016, 6, 23923.

136 M. Pagani, G. Högberg, D. Salmaso, B. Tarnell, A. SanchezCrespo, J. Soares, A. Åberg-Wistedt, H. Jacobsson, T. Hällström and S. A. Larsson, Eur. Arch. Psychiatry Clin. Neurosci., 2005, 255, 359-365.

137 R. J. Lindauer, J. Booij, J. B. Habraken, H. B. Uylings, M. Olff, I. V. Carlier, G. J. den Heeten, B. L. van Eck-Smit and B. P. Gersons, Biol. Psychiatry, 2004, 56, 853-861.

138 O. Bonne, A. Gilboa, Y. Louzoun, D. Brandes, I. Yona, H. Lester, G. Barkai, N. Freedman, R. Chisin and A. Y. Shalev, Biol. Psychiatry, 2003, 54, 1077-1086.

139 S. Mirzaei, P. Knoll, A. Keck, B. Preitler, E. Gutierrez, H. Umek, H. Köhn and M. Pecherstorfer, Neuropsychobiology, 2001, 43, 260-264.

140 J.-K. Zubieta, J. A. Chinitz, U. Lombardi, L. M. Fig, O. G. Cameron and I. Liberzon, J. Psychiatr. Res., 1999, 33, 259-264.

141 I. Liberzon, S. F. Taylor, R. Amdur, T. D. Jung, K. R. Chamberlain, S. Minoshima, R. A. Koeppe and L. M. Fig, Biol. Psychiatry, 1999, 45, 817-826.

142 J. Lucey, D. C. Costa, G. Adshead, M. P. Deahl, G. Busatto, S. Gacinovic, M. Travis, L. S. Pilowsky, P. J. Ell and I. M. Marks, Br. J. Psychiatry, 1997, 171, 346-350.
143 M. Fujita, S. M. Southwick, C. C. Denucci, S. S. Zoghbi, M. S. Dillon, R. M. Baldwin, A. Bozkurt, A. Kugaya, N. P. L. Verhoeff and J. P. Seibyl, Biol. Psychiatry, 2004, 56, 95-100.

144 J. D. Bremner, R. B. Innis, S. M. Southwick, L. Staib, S. Zoghbi and D. S. Charney, Am. J. Psychiatry, 2000, 157, 1120-1126.

145 Y. A. Chung, S. H. Kim, S. K. Chung, J.-H. Chae, D. W. Yang, H. S. Sohn and J. Jeong, Clin. Neurophysiol., 2006, 117, 637642.

146 N. Sachinvala, H. Von Scotti, M. McGuire, L. Fairbanks, K. Bakst, M. McGuire and N. Brown, J. Nerv. Ment. Dis., 2000, 188, 818-823.

147 T. C. Buckley, E. B. Blanchard and W. T. Neill, Clin. Psychol. Rev., 2000, 20, 1041-1065.

148 S. Kim, I. Lyoo, Y. Lee, J. Kim, M. Sim, S. Bae, H. Kim, J. Y. Lee and D. U. Jeong, Acta Psychiatr. Scand., 2007, 116, 145-153.

149 J. C. Britton, K. L. Phan, S. F. Taylor, L. M. Fig and I. Liberzon, Biol. Psychiatry, 2005, 57, 832-840.

150 L. M. Shin, S. P. Orr, M. A. Carson, S. L. Rauch, M. L. Macklin, N. B. Lasko, P. M. Peters, L. J. Metzger, D. D. Dougherty and P. A. Cannistraro, Arch. Gen. Psychiatry, 2004, 61, 168-176.

$151 \mathrm{~J} . \quad$ D. Bremner, M. Vythilingam, E. Vermetten, S. M. Southwick, T. McGlashan, A. Nazeer, S. Khan, L. V. Vaccarino, R. Soufer and P. K. Garg, Am. J. Psychiatry, 2003, 160, 924-932.

$152 \mathrm{~J} . \quad$ D. Bremner, M. Vythilingam, E. Vermetten, S. M. Southwick, T. McGlashan, L. H. Staib, R. Soufer and D. S. Charney, Biol. Psychiatry, 2003, 53, 879-889.

153 W. E. Semple, P. F. Goyer, R. McCORMICK, B. Donovan, R. F. Muzic Jr, L. Rugle, K. McCutcheon, C. Lewis, D. Liebling and S. Kowaliw, Psychiatry, 2000, 63, 65-74.

154 J. D. Bremner, M. Narayan, L. H. Staib, S. M. Southwick, T. McGlashan and D. S. Charney, Am. J. Psychiatry, 1999, 156, 1787-1795.

155 J. D. Bremner, L. H. Staib, D. Kaloupek, S. M. Southwick, R. Soufer and D. S. Charney, Biol. Psychiatry, 1999, 45, 806-816.

156 L. M. Shin, R. J. McNally, S. M. Kosslyn, W. L. Thompson, S. L. Rauch, N. M. Alpert, L. J. Metzger, N. B. Lasko, S. P. Orr and R. K. Pitman, Am. J. Psychiatry, 1999, 156, 575-584.

157 L. M. Shin, S. M. Kosslyn, R. J. McNally, N. M. Alpert, W. L. Thompson, S. L. Rauch, M. L. Macklin and R. K. Pitman, Arch. Gen. Psychiatry, 1997, 54, 233-241.

158 W. E. Semple, P. F. Goyer, R. McCormick, B. Compton-Toth, E. Morris, B. Donovan, G. Muswick, D. Nelson, M. L. Garnett and J. Sharkoff, Psychiatry Res., Neuroimaging, 1996, 67, 17-28.

159 W. E. Semple, P. Goyer, R. McCormick, E. Morris, B. Compton, G. Muswick, D. Nelson, B. Donovan, G. Leisure and M. Berridge, Biol. Psychiatry, 1993, 34, 115-118.

160 L. M. Shin, C. I. Wright, P. A. Cannistraro, M. M. Wedig, K. McMullin, B. Martis, M. L. Macklin, N. B. Lasko, 
S. R. Cavanagh and T. S. Krangel, Arch. Gen. Psychiatry, 2005, 62, 273-281.

161 X. Protopopescu, H. Pan, O. Tuescher, M. Cloitre, M. Goldstein, W. Engelien, J. Epstein, Y. Yang, J. Gorman and J. LeDoux, Biol. Psychiatry, 2005, 57, 464-473.

162 P. Yang, M.-T. Wu, C.-C. Hsu and J.-H. Ker, Neurosci. Lett., 2004, 370, 13-18.

163 M. Driessen, T. Beblo, M. Mertens, M. Piefke, N. Rullkoetter, A. Silva-Saavedra, L. Reddemann, H. Rau, H. J. Markowitsch and H. Wulff, Biol. Psychiatry, 2004, 55, 603-611.

164 R. A. Lanius, P. C. Williamson, M. Densmore, K. Boksman, R. Neufeld, J. S. Gati and R. S. Menon, Am. J. Psychiatry, 2004, 161, 36-44.
165 T. Hendler, P. Rotshtein, Y. Yeshurun, T. Weizmann, I. Kahn, D. Ben-Bashat, R. Malach and A. Bleich, NeuroImage, 2003, 19, 587-600.

166 R. A. Lanius, P. C. Williamson, J. Hopper, M. Densmore, K. Boksman, M. A. Gupta, R. W. Neufeld, J. S. Gati and R. S. Menon, Biol. Psychiatry, 2003, 53, 204-210.

167 R. A. Lanius, P. C. Williamson, K. Boksman, M. Densmore, M. Gupta, R. W. Neufeld, J. S. Gati and R. S. Menon, Biol. Psychiatry, 2002, 52, 305-311.

168 R. A. Lanius, P. C. Williamson, M. Densmore, K. Boksman, M. A. Gupta, R. Neufeld, J. S. Gati and R. S. Menon, Am. J. Psychiatry, 2001, 158, 1920-1922.

169 S. L. Rauch, P. J. Whalen, L. M. Shin, S. C. McInerney, M. L. Macklin, N. B. Lasko, S. P. Orr and R. K. Pitman, Biol. Psychiatry, 2000, 47, 769-776. 